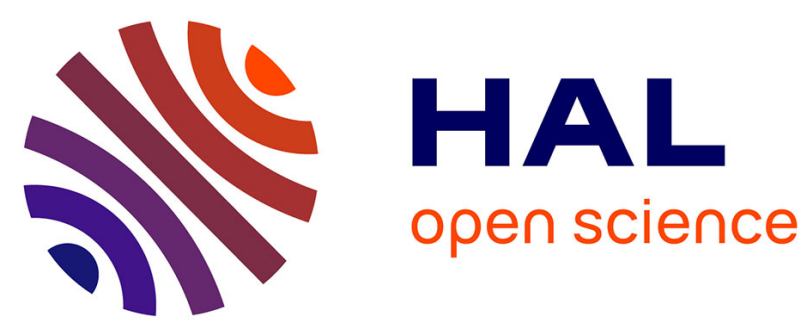

\title{
Observations of Comet 9P/Tempel 1 around the Deep Impact event by the OSIRIS cameras onboard Rosetta
}

Horst Uwe Keller, Michael Küppers, Sonia Fornasier, Pedro J. Gutiérrez, Stubbe F. Hviid, Laurent Jorda, Jörg Knollenberg, Stephen C. Lowry, Miriam Rengel, Ivano Bertini, et al.

\section{To cite this version:}

Horst Uwe Keller, Michael Küppers, Sonia Fornasier, Pedro J. Gutiérrez, Stubbe F. Hviid, et al.. Observations of Comet 9P/Tempel 1 around the Deep Impact event by the OSIRIS cameras onboard Rosetta. Icarus, 2007, 187 (1), pp.87-103. 10.1016/j.icarus.2006.09.023 . insu-03586504

\section{HAL Id: insu-03586504 https://hal-insu.archives-ouvertes.fr/insu-03586504}

Submitted on 23 Feb 2022

HAL is a multi-disciplinary open access archive for the deposit and dissemination of scientific research documents, whether they are published or not. The documents may come from teaching and research institutions in France or abroad, or from public or private research centers.
L'archive ouverte pluridisciplinaire HAL, est destinée au dépôt et à la diffusion de documents scientifiques de niveau recherche, publiés ou non, émanant des établissements d'enseignement et de recherche français ou étrangers, des laboratoires publics ou privés. 


\title{
Observations of Comet 9P/Tempel 1 around the Deep Impact event by the OSIRIS cameras onboard Rosetta
}

\author{
Horst Uwe Keller ${ }^{\text {a }}$, Michael Küppers ${ }^{\mathrm{a}, *}$, Sonia Fornasier ${ }^{\mathrm{b}}$, Pedro J. Gutiérrez ${ }^{\mathrm{c}}$, Stubbe F. Hviid ${ }^{\mathrm{a}}$, \\ Laurent Jorda $^{\mathrm{d}}$, Jörg Knollenberg ${ }^{\mathrm{e}}$, Stephen C. Lowry ${ }^{\mathrm{f}}$, Miriam Rengel ${ }^{\mathrm{a}}$, Ivano Bertini ${ }^{\mathrm{b}}$, \\ Gabriele Cremonese ${ }^{\mathrm{g}}$, Wing-H. Ip ${ }^{\mathrm{h}}$, Detlef Koschny ${ }^{\mathrm{i}}$, Rainer Kramm ${ }^{\mathrm{a}}$, Ekkehard Kührt ${ }^{\mathrm{e}}$, \\ Luisa-Maria Lara ${ }^{\mathrm{c}}$, Holger Sierks ${ }^{\mathrm{a}}$, Nicolas Thomas ${ }^{\mathrm{j}}$, Cesare Barbieri ${ }^{\mathrm{b}}$, Philippe Lamy ${ }^{\mathrm{d}}$, \\ Hans Rickman ${ }^{\mathrm{k}}$, Rafael Rodrigo ${ }^{\mathrm{c}}$, Michael F. A'Hearn ${ }^{1}$, Francesco Angrilli ${ }^{\mathrm{m}}$, \\ Maria-Antonella Barucci ${ }^{n}$, Jean-Loup Bertaux ${ }^{\circ}$, Vania da Deppo ${ }^{\mathrm{p}, q}$, Björn J.R. Davidsson ${ }^{\mathrm{k}}$, \\ Mariolino de Cecco $^{\mathrm{m}, \mathrm{r}}$, Stefano Debei ${ }^{\mathrm{r}}$, Marco Fulle ${ }^{\mathrm{s}}$, Fritz Gliem ${ }^{\mathrm{t}}$, Olivier Groussin ${ }^{1}$, \\ José J. Lopez Moreno ${ }^{c}$, Francesco Marzari ${ }^{u}$, Giampiero Naletto ${ }^{q}$, Lola Sabau ${ }^{v}$, \\ Angel Sanz Andrés ${ }^{w}$, Klaus-Peter Wenzel ${ }^{\mathrm{i}}$
}

\begin{abstract}
The OSIRIS cameras on the Rosetta spacecraft observed Comet 9P/Tempel 1 from 5 days before to 10 days after it was hit by the Deep Impact projectile. The Narrow Angle Camera (NAC) monitored the cometary dust in 5 different filters. The Wide Angle Camera (WAC) observed through

filters sensitive to emissions from $\mathrm{OH}, \mathrm{CN}, \mathrm{Na}$, and $\mathrm{OI}$ together with the associated continuum. Before and after the impact the comet showed regular variations in intensity. The period of the brightness changes is consistent with the rotation period of Tempel 1 . The overall brightness of Tempel 1 decreased by about 10\% during the OSIRIS observations. The analysis of the impact ejecta shows that no new permanent coma structures were created by the impact. Most of the material moved with $\sim 200 \mathrm{~m} \mathrm{~s}^{-1}$. Much of it left the comet in the form of icy grains which sublimated and fragmented within the first hour after the impact. The light curve of the comet after the impact and the amount of material leaving the comet $\left(4.5-9 \times 10^{6} \mathrm{~kg}\right.$ of water ice and a presumably larger amount of dust) suggest that the impact ejecta were quickly accelerated by collisions with gas molecules. Therefore, the motion of the bulk of the ejecta cannot be described by ballistic trajectories, and the validity of determinations of the density and tensile strength of the nucleus of Tempel 1 with models using ballistic ejection of particles is uncertain.
\end{abstract}

(c) 2006 Elsevier Inc. All rights reserved. 


\section{Introduction}

The goal of the Deep Impact mission was to investigate the interior of a comet. When the $\sim 370 \mathrm{~kg}$ projectile hit Comet $9 \mathrm{P} /$ Tempel 1 (hereafter Tempel 1), the Deep Impact spacecraft observed the impact event and the ejecta cloud with high spatial and temporal resolution (A'Hearn et al., 2005). A world-wide campaign of Earth- and space-based observations was initiated to remotely observe the global structure, the composition, and the mid- and long-term evolution of the impact ejecta (Meech et al., 2005).

Among the remote observatories studying Tempel 1, the Rosetta spacecraft, on its way to Comet 67P/ChuryumovGerasimenko, was located particularly well: It was closer to the comet than Earth-based observers (0.53 AU vs $0.89 \mathrm{AU})$ and it was able to observe the comet continuously for more than two weeks. The solar elongation of Tempel 1 of slightly more than 90 deg minimized straylight problems for remote sensing instruments.

OSIRIS is the scientific camera system on Rosetta (Keller et al., 2006). It operates from the near ultraviolet to the near infrared spectral range (about 245-1000 $\mathrm{nm}$ ) and consists of a narrow angle camera (NAC) and a wide angle camera (WAC). The NAC is equipped with 11 medium band width filters (50$90 \mathrm{~nm}$ FWHM) which are designed for the study of the physical properties and the mineralogy of the surface of Comet Churyumov-Gerasimenko with high spatial resolution. The WAC is used with two broad band filters (red and green) and 12 narrow band filters (4-14 nm FWHM). Seven of the narrow band filters isolate gas emissions from the cometary coma; the others measure the dust continuum at wavelengths close to that of the gas emissions.

When the opportunity arose to observe Comet Tempel 1 with OSIRIS, an observing program was designed which necessarily had to be different from that for the in situ study of Comet Churyumov-Gerasimenko. The NAC was used in 4 different filters in the red and near infrared spectral region to monitor the dust coma of Tempel 1 as well as the structure and the color of the impact-created ejecta cloud. During the first $\sim 1.5 \mathrm{~h}$ after the impact, the NAC was effectively used as a photometer that measured the light curve of the inner coma, constraining the processes following the impact. Additional images with a clear filter were taken to be prepared for the case that the effects of the impact on coma brightness would be subtle. The WAC was used to measure the gas production of the comet and to esti- mate the gas content of the material ejected from the nucleus. Emissions from the $\mathrm{OH}$ radical and from atomic oxygen were used as tracers of water production, and the $\mathrm{CN}$ production was measured to compare relative abundances between the impact ejecta and the coma created by normal activity of the comet. Sodium D-line emission was looked for to search for signatures of sodium which may be desorbed from warm dust ejected by the impact.

First results from the OSIRIS observations were reported in Küppers et al. (2005) and Keller et al. (2005). The number of water molecules produced in the impact as determined from measurements of the $\mathrm{OH}$ emission together with estimates of the dust mass derived from the light curve suggested a dust/ice ratio substantially larger than one for the impact ejecta. This would imply that comets are icy dirtballs (Keller, 1989) rather than dirty snowballs (Whipple, 1950). However, some Earthbased observers derived substantially lower dust masses in the ejecta cloud (Harker et al., 2005; Sugita et al., 2005), so that the dust/ice ratio is still under debate. The abundance ratio between the $\mathrm{CN}$ parent molecule(s) and water was somewhat larger in the cloud ejected from the impact than during normal cometary activity. It is, however, not clear yet if this is due to a genuine difference between the surface and the interior of a comet (or between an active and an inactive region) or due to creation of additional $\mathrm{CN}$ from organic material which is more likely to happen during the violent impact than during the normal, more gentle activity of the comet driven by sunlight.

The light curve seen by OSIRIS showed a steep increase in the first $40 \mathrm{~min}$ and then leveled off. The brightness increase, also measured by Earth-based observers (Meech et al., 2005; Schleicher et al., 2006), is much longer than that expected for crater formation. It is also longer than estimates of the time it takes for the ejecta to become optically thin. The probable cause of the long-lasting brightness increase is ejection of material from the impact crater in the form of icy grains. The ice is subsequently sublimated by sunlight, and the grains fragment and the associated increase in cross section causes brightening of the coma. That interpretation is supported by observations of icy grains from the Deep Impact spacecraft (Sunshine et al., 2006). Ejection of icy grains also explains the expansion velocities of the dust in the ejecta cloud, typically $160 \mathrm{~m} \mathrm{~s}^{-1}$ and up to $>400 \mathrm{~ms}^{-1}$, much larger than typical velocities of crater ejecta (Housen et al., 1983). After sublimation of the ice, the dust is accelerated by collisions with the water molecules. 
This paper describes the full data set of OSIRIS observations. We present light curves and color information derived from observations with different filters of the NAC. Coma structures are analyzed and provide information about the interaction between the impact ejecta and the existing coma of the comet. Analysis of the light curves constrains the events during the first minutes after the impact and gives insight into the velocity of the dust. We model the motion of the ejecta cloud with a Monte Carlo code to derive the velocity distribution of the dust and to constrain its size (Jorda et al., this issue). We further present the detection of atomic sodium in the first $\sim 7 \mathrm{~h}$ after the impact, providing clues about the dust ejected by the impact. We revisit the determination of the water and $\mathrm{CN}$ production and provide revised values using the full data set. We discuss implications for the events following the impact and the structure of Comet Tempel 1.

Section 2 summarizes the observations and describes the data reduction. The results are presented in Section 3. Implications for the impact process and the structure of the cometary surface and interior are discussed in Section 4.

\section{Observations and data reduction}

\subsection{Instruments and observing geometry}

The OSIRIS cameras are unobstructed mirror systems with focal lengths of $72 \mathrm{~cm}$ (NAC) and $14 \mathrm{~cm}$ (WAC). The focal ratios are $f / D=8$ for the NAC and $f / D=5.6$ for the WAC. Both cameras are equipped with two filter wheels, containing eight positions each. While the WAC is always operated with a filter in one filter wheel and the other filter wheel in the empty position (no filter), observations with the NAC allow numerous combinations of the 11 medium bandwidth filters, three far focus plates, one near focus plate (for observations of Comet Churyumov-Gerasimenko from distances $\leqslant 2 \mathrm{~km}$ ), and a neutral density filter. The cameras are equipped with backside illuminated CCD detectors comprising $2048 \times 2048$ pixels with a pixel size of $13.5 \mu \mathrm{m}$. The corresponding spatial scales of NAC and WAC are $3.88 \mathrm{arcsec} /$ pixel $(1500 \mathrm{~km} / \mathrm{pixel}$ at Comet Tempel 1 on 4 July) and $20.5 \mathrm{arcsec} / \mathrm{pixel}(7800 \mathrm{~km} / \mathrm{pixel}$ at Comet Tempel 1), respectively. OSIRIS is described in detail by Keller et al. (2006).

Table 1 provides the geometry between Tempel 1, Rosetta, and the Sun for 4 July 2005 at 6 UT, close to the impact time. The relatively close position of Rosetta to the comet, the high solar elongation, and the phase angle of Rosetta which is markedly different from that of Earth-based observers were all favorable for camera observations of Tempel 1 around Deep Impact.

\subsection{The data set}

OSIRIS observations of Comet Tempel 1 started on 28 June 2005 at $23^{\mathrm{h}} 34^{\mathrm{m}}$ UT and ended on 12 July 2005 at $13^{\mathrm{h}} 00^{\mathrm{m}}$ UT. A total of 2058 images of the comet were taken. The Rosetta spacecraft tracked the comet, allowing for long exposure times of up to $30 \mathrm{~min}$.

Table 2 gives the properties of the filter combinations used to image the comet. The WAC was used to image gas emissions and the neighboring dust continuum with filters of 4-10 nm bandwidth. Additionally, the comet was imaged in the broadband red filter (transmission curve relatively close to standard

Table 1

Observing geometry for 4 July 2005 at 6 UT

\begin{tabular}{lllllll}
\hline $\begin{array}{l}\Delta \\
{[\mathrm{AU}]^{\mathrm{a}}}\end{array}$ & $\begin{array}{l}r \\
{[\mathrm{AU}]}\end{array}$ & $\begin{array}{l}\text { Phase angle } \\
{[\mathrm{deg}]}\end{array}$ & $\begin{array}{l}\text { Solar elongation } \\
{[\mathrm{deg}]}\end{array}$ & $\begin{array}{l}\text { "Stereo angle" Earth- } \\
\text { comet-Rosetta }[\mathrm{deg}]\end{array}$ & $\begin{array}{l}\text { Image scale } \\
\mathrm{NAC}[\mathrm{km} / \mathrm{pixel}]\end{array}$ & $\begin{array}{l}\text { Image scale WAC } \\
{[\mathrm{km} / \mathrm{pixel}]}\end{array}$ \\
\hline 0.531 & 1.506 & 69 & 91 & 27 & 1500 & 7800 \\
\hline
\end{tabular}

a Distance between Rosetta and Comet 9P/Tempel 1.

Table 2

Filter combinations used during the OSIRIS observations of Deep Impact

\begin{tabular}{|c|c|c|c|c|}
\hline Camera & Filter(s) & Central wavelength [nm] & Bandwidth (FWHM) [nm] & Remarks \\
\hline \multirow[t]{4}{*}{ NAC } & FFP-vis ${ }^{\mathrm{a}}+$ orange & 647.5 & 86.9 & \\
\hline & FFP-vis ${ }^{a}+$ red & 743.5 & 64.2 & \\
\hline & Near-IR + FFP-IR ${ }^{b}$ & 882.0 & 66.0 & \\
\hline & FFP-vis ${ }^{a}+$ FFP-IR $^{b}$ & $\sim 620^{d}$ & $\sim 470^{\mathrm{d}}$ & Clear filter \\
\hline \multirow[t]{5}{*}{ WAC } & $\mathrm{OH}$ & 308.6 & 3.9 & \\
\hline & $\mathrm{Na}$ & 589.4 & 4.8 & \\
\hline & OI & 630.6 & 4.2 & \\
\hline & Vis 610 & 611.2 & 9.9 & Continuum for $\mathrm{Na}$ and $\mathrm{OI}$ \\
\hline & $\mathrm{R}$ & 627.4 & 155.9 & Red broadband \\
\hline
\end{tabular}

a Re-focussing plate for the visual wavelength range.

b Re-focussing plate for the near-infrared wavelength range.

c Effective wavelength of instrument $\sim 980 \mathrm{~nm}$ due to variation of CCD quantum efficiency over filter bandpass.

${ }^{\mathrm{d}}$ Short wavelength cut-off due to FFP-IR becoming opaque, long wavelength cut-off due to decrease in CCD quantum efficiency. 
Table 3

Overview of the OSIRIS observations of Comet Tempel 1

\begin{tabular}{|c|c|c|c|c|c|c|}
\hline Camera & Filter & Phase & $\begin{array}{l}\text { Approximate repetition } \\
\text { frequency }\end{array}$ & $\begin{array}{l}\text { Exposure } \\
\text { time }[s]\end{array}$ & $\begin{array}{l}\text { CCD window } \\
\text { size [pixels] }\end{array}$ & $\begin{array}{l}\text { CCD binning } \\
\text { factor }\end{array}$ \\
\hline \multirow[t]{5}{*}{$\mathrm{NAC}$} & FFP-vis + orange & Monitoring & $1.5 \mathrm{~h}$ & 600 & $320 \times 320^{\mathrm{a}}$ & 1 \\
\hline & FFP-vis + red & Monitoring & $1.5 \mathrm{~h}$ & 900 & $320 \times 320^{\mathrm{a}}$ & 1 \\
\hline & $\mathrm{IR}+\mathrm{FFP}-\mathrm{IR}$ & Monitoring & $3 \mathrm{~h}$ & 1800 & $320 \times 320^{\mathrm{a}}$ & 1 \\
\hline & FFP-vis + FFP-IR & Monitoring & $3 \mathrm{~h}$ & 300 & $320 \times 320^{\mathrm{a}}$ & 1 \\
\hline & FFP-vis + orange & Impact & $47-85 \mathrm{~s}$ & 45 & $320 \times 320$ & 1 \\
\hline \multirow[t]{6}{*}{ WAC } & $\mathrm{OH}$ & Monitoring & $3 \mathrm{~h}$ & $2 \times 600$ & $128 \times 128$ & 1 and $4^{b}$ \\
\hline & $\mathrm{CN}$ & Monitoring & $3 \mathrm{~h}$ & $2 \times 600$ & $128 \times 128$ & 1 and $4^{b}$ \\
\hline & UV 375 & Monitoring & $3 \mathrm{~h}$ & $2 \times 600$ & $128 \times 128$ & 1 and $4^{b}$ \\
\hline & $\mathrm{Na}$ & Monitoring & $3 \mathrm{~h}$ & 1200 & $128 \times 128$ & 1 \\
\hline & OI & Monitoring & $3 \mathrm{~h}$ & 1200 & $128 \times 128$ & 1 \\
\hline & Vis 610 & Monitoring & $3 \mathrm{~h}$ & 1200 & $128 \times 128$ & 1 \\
\hline
\end{tabular}

a Single exposure covering $512 \times 512$ pixels at the end of the monitoring campaign.

b Unbinned exposures and binning $4 \times 4$ were used alternately ( 2 unbinned exposures, 2 binned exposures $3 \mathrm{~h}$ later, and so on).

$\mathrm{R}$ filters) in order to look for large scale coma structures. From the medium bandwidth filters of the NAC, 4 filters in the red and near infrared spectral range were chosen to image the cometary dust with as little gas contamination as possible. Additional images with a clear filter (combination of visual and infrared focus plate) were taken for maximum signal/noise.

The observations are divided into a near-impact phase $\left(5^{\mathrm{h}} 34^{\mathrm{m}}\right.$ to $7^{\mathrm{h}} 19^{\mathrm{m}}$ on 4 July 2005 , with the signal from the impact having been received at $T=5^{\mathrm{h}} 49^{\mathrm{m}} 01^{\mathrm{s}}$ at Rosetta) and a monitoring phase, which covers everything outside the impact phase. To accommodate a request by the ultraviolet spectrometer ALICE, most of the monitoring phase was divided into blocks of $3 \mathrm{~h}$ duration. Within each block, the spacecraft pointed at 5 different positions, with the most distant positions being 11 arcmin apart. As a consequence, the position of the comet on the OSIRIS CCDs varied with time. During the impact phase, the same sequence of pointing positions was used, but the pointing duration on each position decreased. At the end of the monitoring on 14 July 2005, the spacecraft kept the pointing constant for roughly $10.5 \mathrm{~h}$.

OSIRIS observations during the monitoring phase were interrupted between 29 June $4^{\mathrm{h}}$ UT and 30 June $14^{\mathrm{h}} 34^{\mathrm{m}}$ due to an anomaly of the OSIRIS software. Further interruptions of $3 \mathrm{~h}$ each took place on 2 July at $5^{\mathrm{h}} 34^{\mathrm{m}}$, on 7 July at $1^{\mathrm{h}} 19^{\mathrm{m}}$, and on 12 July at $1^{\mathrm{h}} 19^{\mathrm{m}}$ to accommodate off-loadings of the reaction wheels of the spacecraft. Nevertheless, the nearly continuous coverage during more than two weeks makes this data set unique. Table 3 shows the image parameters for each filter during both the monitoring phase and the impact phase.

\subsection{Data reduction}

\subsubsection{Image calibration}

The OSIRIS CCDs are read-out using an intricate dual 14bit amplifier/ADC (Analogue to Digital Converter) chain which is combined to an effective 16-bit system. The ADC delivers $\sim 3 e^{-}$per Digital Unit (DN). To convert the ADC output into physical units the image data are passed through a standard image calibration pipeline. The pipeline performs the following steps: (a) First the digital offset of the two ADC chains is removed (DN values above $2^{14}-1$ get an additional offset added by the readout electronics to clearly separate data processed by the two ADC channels). (b) The image data are passed through a coherent noise filtering routine that filters out the noise coming from the power converter. (c) The bias offset is removed using either calibration data acquired during the observational campaign or using database values (the Deep Impact data were calibrated using calibration data from the campaign). (d) The image data are converted into $\mathrm{DN} \mathrm{s}^{-1}$ by dividing the image by the exposure time. (e) The image data are passed through a dark current removal routine. The OSIRIS CCDs are operated at a very low temperature (NAC at $\sim 150 \mathrm{~K}$ and WAC at $\sim 170 \mathrm{~K})$. At this temperature the average dark current production is a small fraction of a DN. The only dark current correction performed on the OSIRIS images is removal of a small "warm" area at the center of the NAC CCD. (f) Bad pixels are filtered out. Currently the only bad pixels on the OSIRIS CCDs are in a single "warm" column on the NAC CCD. (g) The images are flat fielded using reference flat fields from the ground calibration. (h) Finally the digital data are converted into physical values using a conversion factor derived from observations of Vega, secondary spectrophotometric standard stars (58 Aql and $\varepsilon$ Aqr), and the solar analogs 16 Cyg A and 16 Cyg B. For the early type stars, the conversion factor is converted into that for a solar input spectrum using knowledge of the quantum efficiency of the $\mathrm{CCD}$, the optical response of the telescope, and the optical filter transmissions. The output of the calibration pipeline is in units of $\left[\mathrm{W} \mathrm{m}^{-2} \mathrm{sr}^{-1} \mathrm{~nm}^{-1}\right]$. 


\subsubsection{Extraction of light curves for gaseous emissions}

The radiometrically calibrated data were used as input for the computation of the WAC light curves in the gas filters. Because of the low signal/noise in some of the filters, the exact position of the comet could not always be easily identified in the images. Therefore, the following approach has been used to determine the actual position of the comet in a given image. First, the spacecraft pointing information provided by ESA was used to compute the nominal position of the comet at the time the image was acquired. Then, this nominal position was compared to the brightness maximum in the broadband continuum images taken with the red filter where the signal/noise ratio was sufficiently high. Under the assumption that the nucleus position (determined with sub pixel accuracy) could well be approximated by the red filter brightness maximum, a time dependent offset correction was computed and the corresponding shift applied to the image. Another problem inherent in the OSIRIS data was the contamination of the images by cosmic rays and stars inside the aperture. To remove these disturbances as well as possible, a comparison of the actual image with a median stack of 7 images taken around the actual image ( 3 images before and 3 images after) was made, and pixels deviating from the median value by more than 2 standard deviations, as estimated from the width of the histogram of the median stack image, were replaced by the corresponding median stack value. This procedure was applied to the gas filter image as well as to the associated continuum image. Where our observational sequence contained two images closely spaced in time (as was the case for the $\mathrm{CN}$ and $\mathrm{OH}$ filters), additionally the minimum of both images was taken as representative for the time step, thereby again suppressing possible remnants of stars and cosmics. Then the continuum image was multiplied by a correction factor accounting for the difference in the passband between the two filters and subtracted from the original gas filter image. This image together with the information about the comet's position was then used as input to a standard photometry routine, which sums up the signal in the specified apertures around the center and subtracts the contribution of the background sky, estimated by the modal value of the histogram of the sky pixels.

\section{Results}

\subsection{Properties of the dust from NAC images}

The images obtained with the NAC allow us to study the structure, temporal evolution, and color of the dust, both in the cometary coma before the impact and in the dust cloud created by the impact. The signal/noise of individual images, however, is insufficient to study details of the dust distribution in space and time. Therefore, we used two different ways of enhancing the signal/noise: First, we summed up the intensity in individual images over circular "apertures" centered on the nucleus. This method keeps the full time resolution, but sacrifices spatial information. Second, we added groups of images taken within specific time intervals, preserving the spatial resolution at the cost of lower temporal resolution.
Since the filter set of the NAC was not designed for observations of cometary comae from large distances, they do not strictly separate the dust contribution from that of the gas. From a comparison with ground-based spectra (Lara et al,, in preparation), we estimate the contribution of the gas to the signal in the inner coma (within an 8 arcsec circle around the nucleus) in the orange and clear filter to be 4 to $8 \%$. The contribution is less in the red, near-IR, and IR filters. The gas contribution varies with distance from the nucleus, the details depending on the lifetimes of the gas species involved and their parents. Most of the results presented in this section are insensitive to the gas contribution to the observed flux; exceptions will be discussed specifically.

In Section 3.1.1 we analyze the brightness variations of the normal cometary coma unaffected by the impact. In Section 3.1.2 we describe the spatial structure of the impact ejecta, Section 3.1 .3 covers the spatial evolution of the impact cloud on different timescales, and Section 3.1.4 deals with the size and velocity distribution of the dust and constraints on the dust production rate. Finally, Section 3.1.5 derives information about the color of the dust.

\subsubsection{Brightness variations of the cometary coma}

We performed cometocentric circular aperture photometry on the images of Tempel 1. The central pixel or optocenter of the comet was manually determined. We then converted the fluxes measured by OSIRIS to the quantity Af $\rho$ (A'Hearn et al., 1984).

The brightness of the comet in images taken with the orange filter and using an aperture with a radius of $7500 \mathrm{~km}$ (5 pixels) corresponds to values of Af $\rho$ of $99 \pm 2 \mathrm{~cm}$ before impact and $91 \pm 2 \mathrm{~cm}$ about a week after impact. A slight decrease in the flux is also detected within the pre-impact data (covering the time between 29 th of June and 4 th of July). The Af $\rho$ values are $3 \%$ lower than those in Keller et al. (2005) because the flux calibration of OSIRIS has been revised. Af $\rho$ is slightly lower for smaller aperture sizes $(\sim 10 \%$ lower for an aperture radius of $3000 \mathrm{~km}$ ).

The coma background flux was subtracted from the light curve by calculating the median of the pre-impact fluxes for each aperture and filter, and by subtracting each computed median value from all fluxes. The resulting fluxes were integrated over nine circular apertures of different radii, from two to ten pixels ( 1 pixel $=1500 \mathrm{~km}$ ). Since the radius of a one-pixel aperture would be smaller than the Point Spread Function (PSF) (for the Clear filter in the NAC, the PSF it is estimated to have a FWHM of 1.725 pixels $=6.5^{\prime \prime}$ ), light curves at this size are not used in our analysis.

In Fig. 1 we present the lightcurve obtained with the clear filter which shows the best signal/noise ratio. The flux decrease seen in the orange filter is confirmed in our data points well after the impact (10th July and later), where the mean level is approximately $10 \%$ below the mean level of the pre-impact data. This decrease in background flux around the impact was also seen by Earth-based observers (e.g., Schleicher et al., 2006).

To look for flux changes on shorter timescales, we subtracted an exponential fit to the impact ejecta from the lightcurve. We 


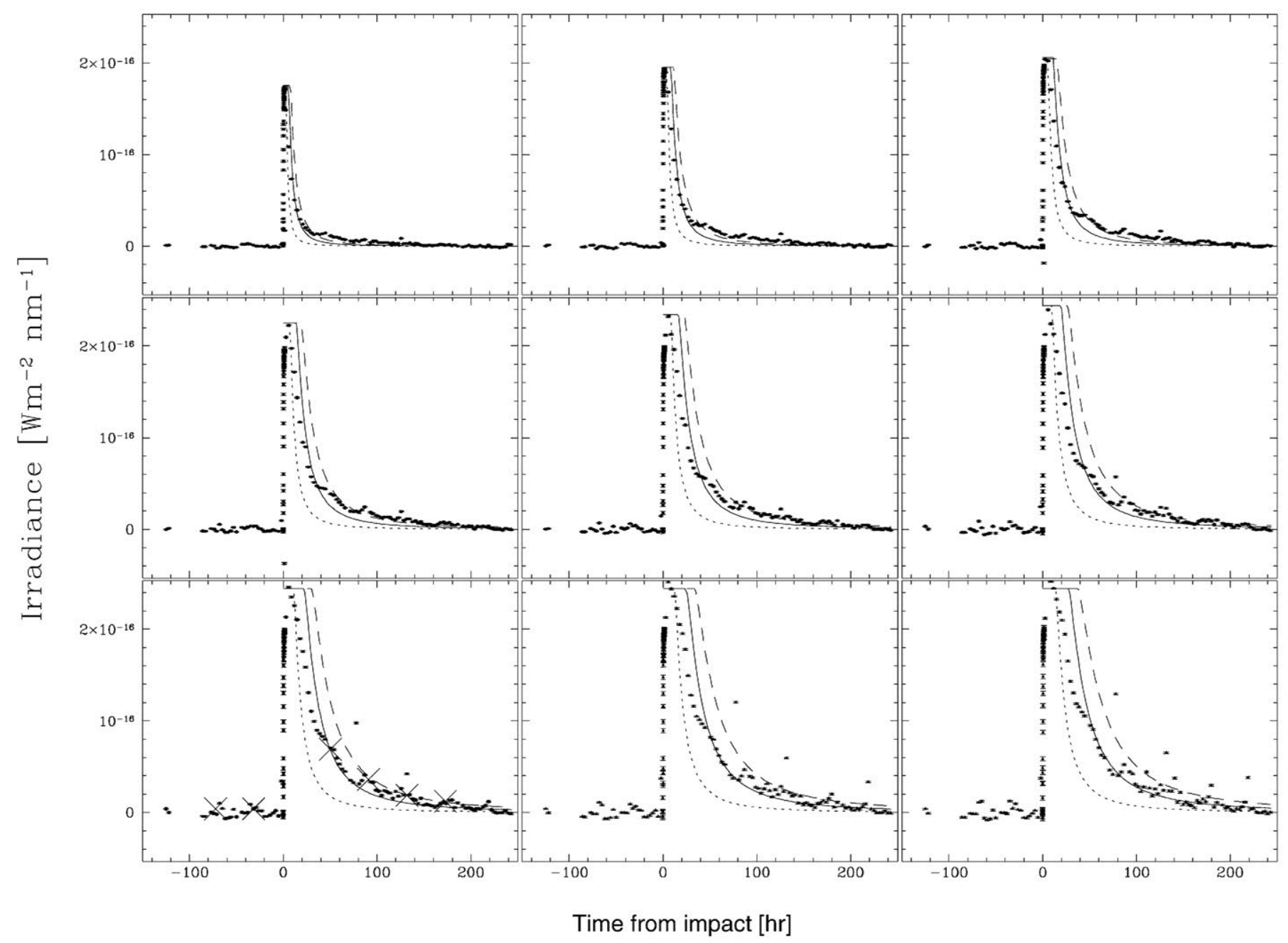

Fig. 1. Light curves of the cometary dust in the clear filter, for nine circular fields from 2 to 10 pixels or 3000 to $15,000 \mathrm{~km}$ radius at the comet (from left to right). The coma subtraction error is estimated to be $\sim 2$ times the error bars. The dashed, solid and dotted lines show model profiles for velocities of 110,160 , and $300 \mathrm{~m} \mathrm{~s}^{-1}$, respectively. The crosses on the bottom left panel are separated by $40.832 \mathrm{~h}$, the rotation period of Comet Tempel 1 .

detect noticeable changes in brightness of $\sim 4 \%$ both pre- and post-impact, also visible in the lightcurves in Fig. 1. The locations of these maxima are repeated approximately each $\sim 41 \mathrm{~h}$, close to the spin period determined by A'Hearn et al. (2005) and Belton et al. (2006).

Fig. 2 shows the data phased with a rotation period of $40.74 \mathrm{~h}$. An aperture with a radius of four pixels $(6000 \mathrm{~km})$ was used. Each rotation period is normalized to its average. It can be seen that the flux variation is strongly correlated with rotation. However, the relative amplitude is less than $\pm 3 \%$. The strong variability seen in observations of the bare nucleus at large heliocentric distance (Belton et al., 2005) is suppressed by superposition with the signal from a largely isotropic dust coma.

\subsubsection{Structure of the impact ejecta and influence of the impact on the structure of the cometary coma}

3.1.2.1. Ejecta plume morphology For each clear-filter image taken with NAC the comet centers were carefully determined, and used to shift the images so that the comet center resided at the same pixel location in each image. The image list was then divided into multiple groups. Within a given group, the images were median combined with median-filtering applied, which efficiently removed all cosmic rays and background stars (with the exception of just one sub-group to be discussed below). Finally the images were trimmed to leave only the usable area of the co-added images (i.e., that part of the images not affected by shifting). For this process we chose to use the NAC images taken with the clear filter to optimize the spatial resolution and signal/noise of the resulting co-added images. By grouping the images we lose temporal resolution but gain signal/noise in order to bring up fainter plume structures.

Table 4 lists the average UT dates of the various groups used in the plume-evolution analysis, along with the individual image exposure times, effective exposure times of the co-added images, and the time relative to impact in hours.

In Fig. 3 we show black-and-white images of eight co-added groups. The upper left panel is a pre-impact image $(T-7.25 \mathrm{~h})$. Distortions in the coma are clearly seen from $T+13.23 \mathrm{~h}$ 


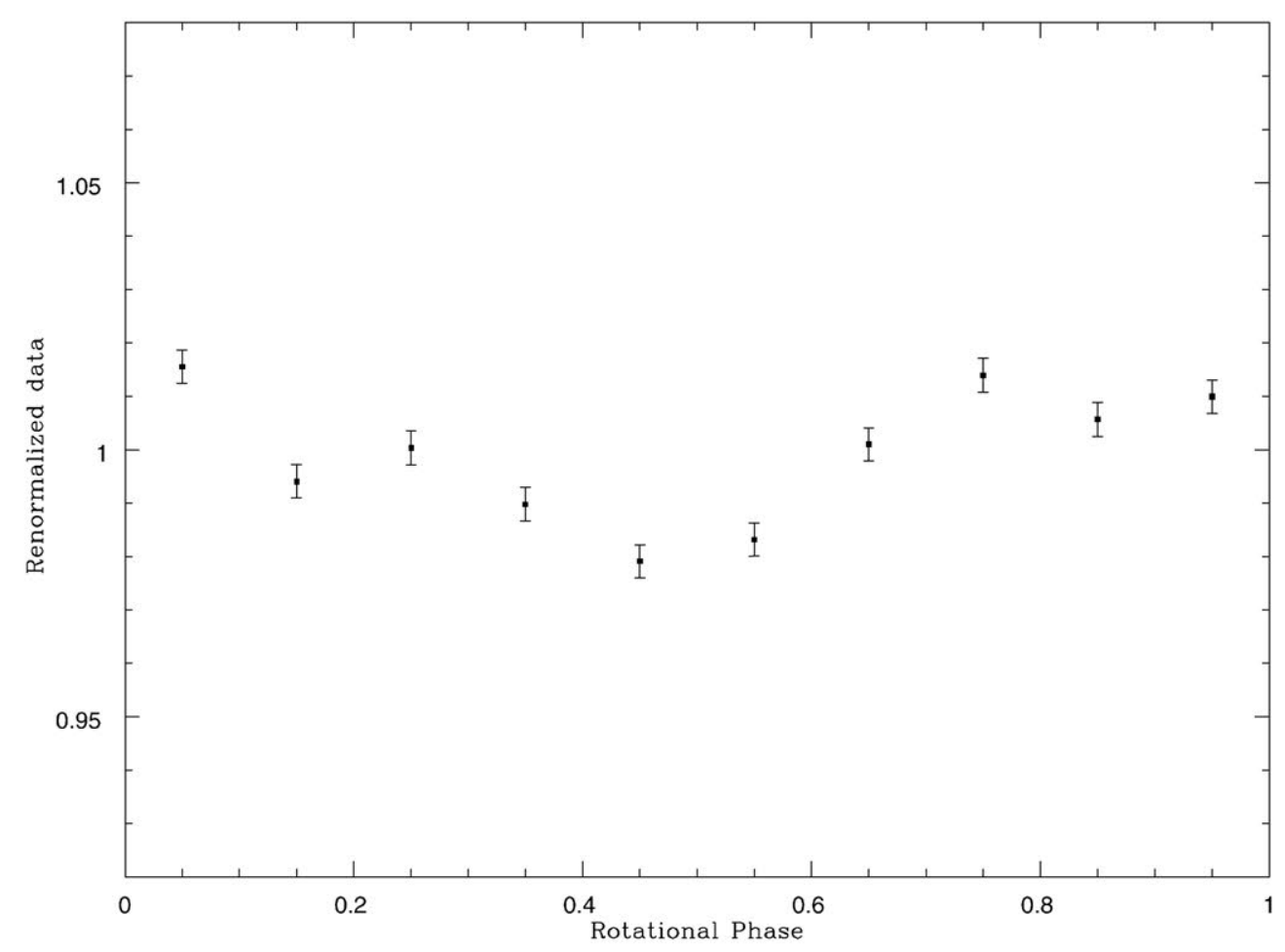

Fig. 2. Brightness variation in an aperture with a radius of 4 pixels $(6000 \mathrm{~km})$. All data are normalized to the mean brightness within each rotation period. Data are binned with bin widths of 0.1 in rotational phase.

Table 4

Groups of images used for the analysis of ejecta plume morphology

\begin{tabular}{lccc}
\hline $\begin{array}{l}\text { Average UT date of each group } \\
\text { [day of July 2005] }\end{array}$ & $\begin{array}{l}\text { Individual exp. time } \\
{[\mathrm{s}]}\end{array}$ & $\begin{array}{l}\text { Effective exp. time } \\
{[\mathrm{s}]}\end{array}$ & $\begin{array}{l}\text { Time relative to impact } \\
{[\mathrm{h}]}\end{array}$ \\
\hline 3.9423 & $8 \times 300,6 \times 35$ & 2610 & -7.25 \\
4.2785 & $41 \times 35$ & 1435 & 0.82 \\
4.7955 & $8 \times 300$ & 2400 & 13.23 \\
5.7955 & $8 \times 300$ & 2400 & 37.23 \\
6.7509 & $7 \times 300$ & 2100 & 60.15 \\
7.7955 & $8 \times 300$ & 2400 & 85.23 \\
8.7955 & $8 \times 300$ & 2400 & 109.23 \\
9.7955 & $8 \times 300$ & 2400 & 133.23 \\
10.7955 & $8 \times 300$ & 2400 & 157.23 \\
11.7509 & $7 \times 300$ & 2100 & 180.15 \\
12.7955 & $8 \times 300$ & 2400 & 205.23 \\
13.7953 & $8 \times 300$ & 2400 & 229.23 \\
\hline
\end{tabular}

Note. All images were taken with the clear filter.

onwards, and the comet eventually gets very close to its approximate pre-impact state around $T+133.23 \mathrm{~h}$ (lower right panel).

To monitor the evolution of the ejecta plume we subtract the pre-impact image from each of the post-impact co-added images $(T-7.25 \mathrm{~h})$, to leave just flux from the post impact material. The resultant images are then enhanced using the boxcar smoothing method which involves replacing each pixel value with the average of the surrounding $3 \times 3$-pixel box. The results are shown in Fig. 4, where the evolution of the plume is clearly seen. The material first forms a cloud around the nucleus and is then pushed in the anti-solar direction by solar radiation pressure. Faint remnants of the plume can be seen out to $T+157.23 \mathrm{~h}$. Note that the diagonal (top left to bottom right) low flux-level features in the upper left panel are from the wings of background stars that were not completely removed by the median-filtering process. This was due to the small time separation between frames within that group which reduced the effectiveness of the median filtering. However, this did not affect the analysis.

Some interesting filamentary structure can be seen at $T+$ $13.23 \mathrm{~h}$, and Fig. 5 is a close-up of the near-nucleus region. Some faint curved structures can be seen emanating from the comet in multiple directions (particularly evident by the overplotted contours). The approximate location of these features is marked by dotted lines, with the most prominent being the feature extending into the SW quadrant of the figure. This may be interpreted as some of the ejected material being collimated, 

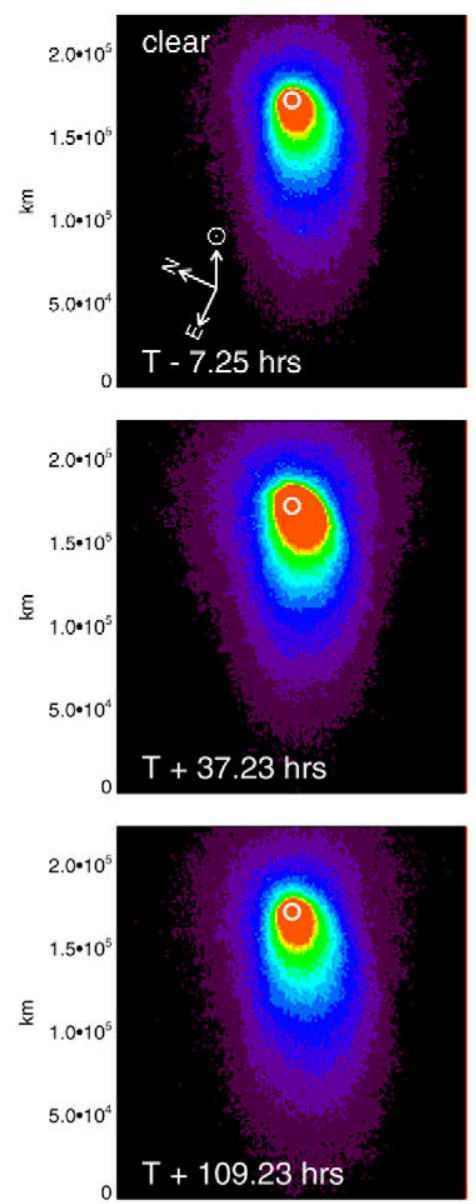

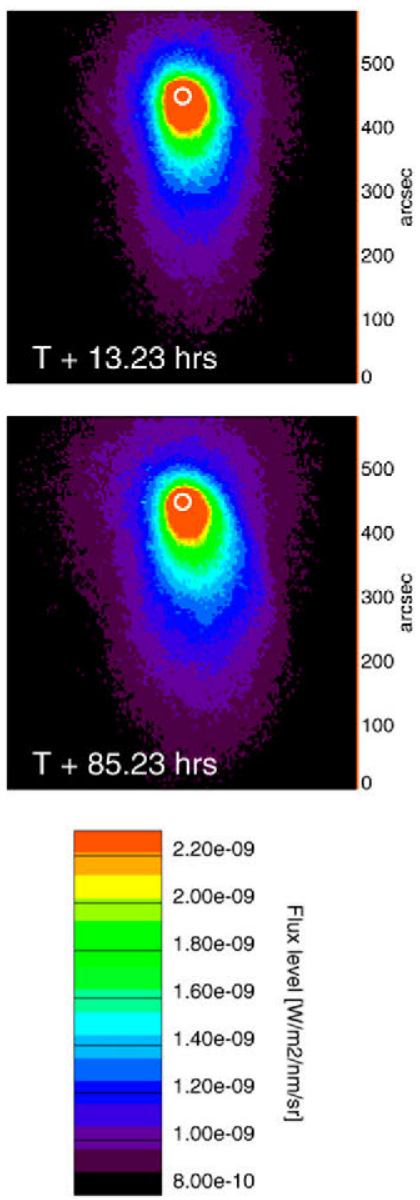

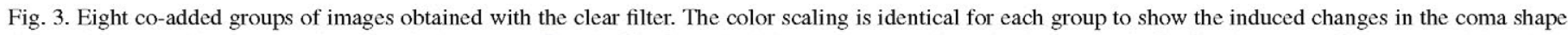




to the impact [h] (i.e., the average UT of each group minus the UT time of impact).

and then quickly dispersed. However, it may also be residuals of the subtraction of the pre-impact coma.

\subsubsection{Coma/jet/tail morphology A search was made for} changes in the coma/tail/jet structure that may have been caused by the impact. This analysis was performed differently than that of the plume morphology. First, the image list was divided up according to the rotation rate of the comet. The comet is known to be rotating at $P \sim 40.8 \mathrm{~h}$, obtained from the Deep Impact spacecraft data (A'Hearn et al., 2005; Belton et al., 2006). Table 5 gives the average UT dates, exposure times and time from impact for each group of images. Each bin was $\sim P / 2 \mathrm{~h}$ long, so that the average UT from the images within a given bin was as close as possible to 0.0 or 0.5 rotational phase. This was done to disentangle, as much as possible, rotation-induced effects on the coma structure from effects caused by the impact. Again, we sacrifice temporal resolution for signal/noise, the latter being far more important when looking for faint jet features than for the plume structures above.

As above, we carefully shifted and median-combined the images within each group. We applied an image analysis method that is specifically designed to look for radial jet-like features embedded in the coma, which presumably originate from the near-nucleus jets. This involves constructing a new image from the original co-added image in the following way. For every azimuth $\theta$ (centered on the nucleus), we measure the radial brightness profile at $\pm \Delta \theta$ deg. The average is measured and is then subtracted from the radial brightness profile at $\theta$. This is repeated at all azimuths. Various images are created for a range of $\Delta \theta$ (i.e., $0^{\circ}-35^{\circ}$ at $2.5^{\circ}$ intervals), but we find that a value around $17.5^{\circ}$ produces good results. The resultant images are boxcar smoothed and can be seen in Fig. 6. This method does not target non-radial features like the dust shells as seen in ground-based imaging (Licandro, 2005). Laplacian filtering, which detects 'edges' in the frames, is better suited for this. These methods were attempted, but unfortunately the sig$\mathrm{nal} /$ noise was too low to be effective.

One can see that the main feature lies along the anti-solar direction, and spans the full length of the tail as seen in the original co-added images. This feature may be the jet described by Schleicher et al. (2006). The direction of this strong single jet does not change significantly with rotational phase, and as noted by Schleicher et al. (2006), most likely implies that the jet originated from a source near the pole.

There are no huge differences in the image structure between the pre- and post-impact processed images. The first two 

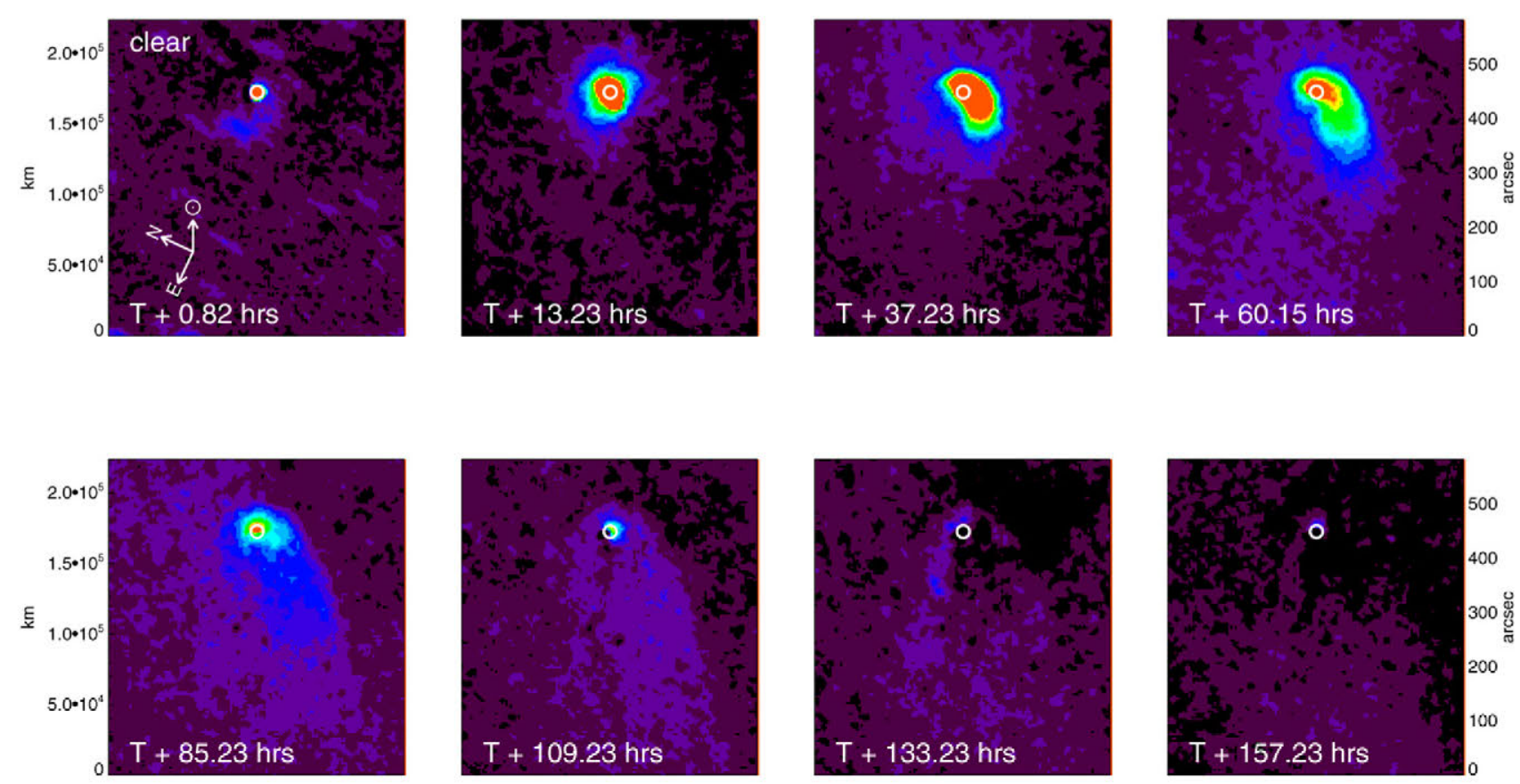

Fig. 4. Images of the ejecta plume. The post-impact images of Fig. 3 divided by the pre-impact image.

panels are from pre-impact images at 0.0 and 0.57 rotational phase, and represent the baseline behavior. The main jet that lies along the antisolar direction appears to increase in intensity from $T+50.73 \mathrm{~h}$ to $T+92.73 \mathrm{~h}$, possibly resulting from ejected material feeding the existing jet. Also, within this time a new filament appears to form in the southern direction (marked with an arrow) and moves outwards, then eventually disperses. This new filament is perhaps a result of newly exposed ices coming back into view of the Sun as the nucleus rotates. It may also be an artifact of the expanding plume which is particularly prominent during this time. In any case, this analysis awaits detailed modeling.

\subsubsection{Formation of the impact cloud}

The basic development of the impact cloud can be seen in Fig. 1: During the first $40 \mathrm{~min}$ after the impact, the flux from the ejecta is continuously increasing, reaching a factor of $\sim 5$ in the aperture with radius 2 pixels $(3000 \mathrm{~km})$. The increase takes longer than the crater formation time (3-6 min, Schultz et al., 2005). During the following $50 \mathrm{~min}$, the brightness leveled off. After $90 \mathrm{~min}$, the flux started to decrease because the material started to leave the aperture (Küppers et al., 2005).

A detailed inspection of the light curve for the first few minutes after the impact (Fig. 7) shows that the slope of the brightness increase changes twice (Keller et al., 2005). A steep increase in the first one or two minutes is followed by a period of stagnation with very little brightness increase. After approximately four minutes the slope increases again.

We compared the brightness increase during the first few minutes with the ejected mass as a function of time as predicted by the Z-model of crater formation (Maxwell, 1977; Croft, 1980; described in Melosh, 1989). The Z-model describes the mass ejected from an impact crater as a function of time. While the Z-model predicts some decrease in mass ejec- tion per time unit, it does not reproduce the stagnation seen in the light curve 2-4 min after the impact. The reason for the discrepancy between model and observations may be our implicit assumption that the relation between flux and ejected mass is linear. It may be non-linear, either because increasing optical depth of the impact ejecta limits the flux from newly produced material or because the size distribution of the ejecta changes with time. If late ejecta are larger than early ones, the cross section per mass unit decreases, resulting in less flux from the same excavated mass.

After $4 \mathrm{~min}$ the lightcurve steepens and then the slope remains approximately constant until $15 \mathrm{~min}$ after the impact. It is likely that the crater excavation has finished before or at the beginning of this phase. The steepening is caused by fragmentation of dust grains and by decreasing optical depth of the expanding ejecta cloud.

The slope of the light curve in the first 15-20 min after the impact shows that the ejected material was quickly accelerated. We estimate the particle expansion velocity as follows: assuming that the signal is created by reflection of sunlight from dust with an albedo of 0.1 , we calculate the total cross section of the material creating the signal from the ejecta cloud. At each time, the material is distributed over an area at least as large as the total cross section seen, and over a much larger area once it becomes optically thin. Therefore the radius of a circle with the cross section of the dust corresponds to the minimum distance traveled by the dust. The increase of this radius per time unit is the minimum dust velocity.

The result is shown in Fig. 8. Allowing $5 \mathrm{~min}$ for crater formation, we calculate a minimum velocity of $5 \mathrm{~m} \mathrm{~s}^{-1}$ to explain the brightness increase between 5 and $15 \mathrm{~min}$ after the impact. This is about a factor of 3 larger than the escape velocity of Comet Tempel 1 (Belton et al., 2005). For an impact dominated by gravity as suggested by A'Hearn et al. (2005), only a negli- 
Table 5

Groups of images used for the analysis of coma and jets

\begin{tabular}{|c|c|c|c|}
\hline $\begin{array}{l}\text { Average UT date of each group } \\
\text { [day of July 2005] }\end{array}$ & $\begin{array}{l}\text { Individual exp. time } \\
{[\mathrm{s}]}\end{array}$ & $\begin{array}{l}\text { Effective exp. time } \\
{[\mathrm{s}]}\end{array}$ & $\begin{array}{l}\text { Time relative to impact } \\
\text { [h] }\end{array}$ \\
\hline 2.9726 & $6 \times 300$ & 1800 & -30.52 \\
\hline 3.9484 & $7 \times 300,4 \times 35$ & 2240 & -7.10 \\
\hline 4.3286 & $41 \times 35,6 \times 300$ & 3235 & 2.02 \\
\hline 5.4830 & $7 \times 300$ & 2100 & 29.73 \\
\hline 6.3580 & $7 \times 300$ & 2100 & 50.73 \\
\hline 7.2538 & $6 \times 300$ & 1800 & 72.23 \\
\hline 8.1080 & $7 \times 300$ & 2100 & 92.73 \\
\hline 8.9205 & $6 \times 300$ & 1800 & 112.23 \\
\hline 9.7330 & $7 \times 300$ & 2100 & 131.73 \\
\hline 10.6080 & $7 \times 300$ & 2100 & 152.73 \\
\hline 11.4830 & $7 \times 300$ & 2100 & 173.73 \\
\hline 12.3997 & $7 \times 300$ & 2100 & 195.73 \\
\hline
\end{tabular}

Note. All images were taken with the clear filter.

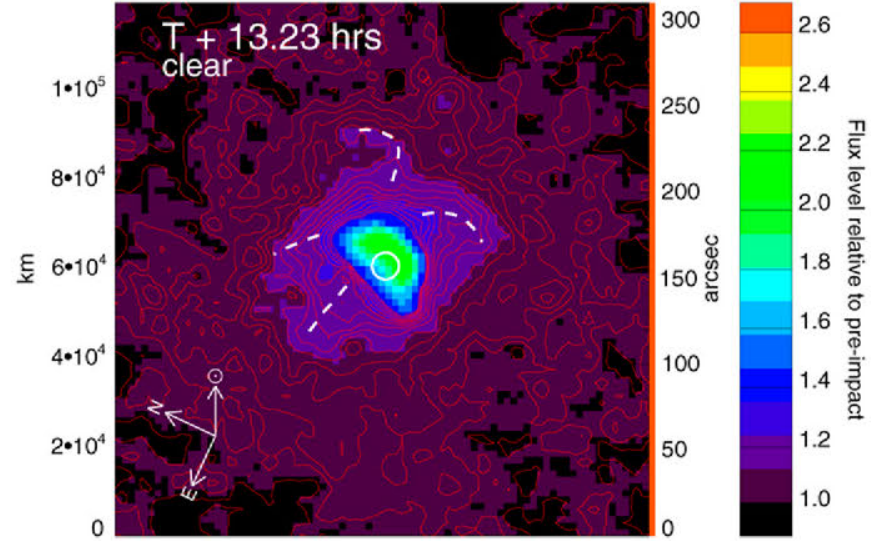

Fig. 5. Close-up image of the near-nucleus region in the second panel of Fig. 4. The color scaling has been adjusted slightly from Fig. 4, and contours have been overplotted for clarity. The dotted lines indicate the approximate location of features interpolated as structures in the impact ejecta.

gible fraction of the impact ejected mass reaches that velocity (Housen et al., 1983). Following Housen et al.'s scaling laws, a strength of about $5 \times 10^{4} \mathrm{~Pa}$ is needed in order to have $10 \%$ of the ejecta reach $5 \mathrm{~m} \mathrm{~s}^{-1}$ or more. This strength is larger than that considered by advocates of a possible strength-controlled cratering event during Deep Impact (Holsapple and Housen, 2006). Our lower limit for the velocity implies that the acceleration of the impact ejecta by cometary gas (either the background coma or from sublimation of icy grains among the ejecta) started in the first minutes after the impact and close to the nucleus.

The long-term evolution of the ejecta is shown in Fig. 9. It shows the total flux from the image groups described in Section 3.1.2. Additionally, a similar analysis is performed for images taken with the orange filter. An apparent increase of $\sim 10 \%$ is seen until $60 \mathrm{~h}$ after the impact, then the observed flux decreases sharply. It is tempting to interpret the flux increase as continued dust production days after the impact or fragmentation of dust particles. However, an alternative explanation is the contribution of fluorescence emission from gas to the flux. Immediately after the impact, only parent molecules were present in the impact cloud. On a timescale from hours to days (details depend on the lifetime of each molecule against solar radiation), the radicals observed in the visible range first are produced by dissociation of their parent molecules and then dissociate or ionize themselves. The steady-state contribution of $4-8 \%$ to the signal in the orange and clear filter over a limited spatial range may be consistent with a $10 \%$ contribution for a limited time during the evolution of the cloud.

\subsubsection{Ejecta velocity and size distribution, dust production by the impact}

A first estimate of the ejecta velocity comes from the decay of the flux when the material leaves the different apertures seen in Fig. 1 (Keller et al., 2005; Küppers et al., 2005). Typical velocities derived this way are between 100 and $300 \mathrm{~m} \mathrm{~s}^{-1}$. As an illustration, Fig. 1 shows model lightcurves for particles with projected velocities of 110,160 and $300 \mathrm{~m} \mathrm{~s}^{-1}$. The velocity estimates do not vary with the size of the aperture, although we assume that the dust reaches its final velocity instantly at the time of impact. Dust with a velocity of $200 \mathrm{~m} \mathrm{~s}^{-1}$ reaches the edge of the smallest aperture (3000-km or 2-pixel radius) in slightly more than $4 \mathrm{~h}$. The fact that we do not see the acceleration phase (as an apparently lower velocity in smaller apertures) implies that we that not more than a few percent of the impact related material was produced later than $1 \mathrm{~h}$ after the impact. Also, the acceleration of the dust took place within the first hour. No impact ejecta are seen after $\sim 157 \mathrm{~h}$ in any aperture, primarily due to the radiation pressure removing the material.

In order to get a more accurate estimate on the size-velocity distribution of the dust, we used a Monte Carlo model to reproduce the motion of the ejecta cloud under the influence of radiation pressure. We analyzed five NAC images taken between 1.42 and 3.73 days post-impact obtained with the orange filter in order to estimate the mass of the refractory dust particles excavated during the impact.

Our method works in three steps. First of all, we create a grid of synthetic images corresponding to given bins of dust sizes and velocities. We account for the three-dimensional geometry of the dust emission and we take into account the radiation pressure using Mie theory. The images are calibrated in flux using Mie coefficients integrated over the bandpass of the orange filter. We then constrain the maximum velocity allowed 



Fig. 6. Co-added groups of images with the radial profile subtracted. The arrows in the lower panels indicate the location of the only feature that appears to evolve with time. It is unknown whether or not this feature is related to the impact.

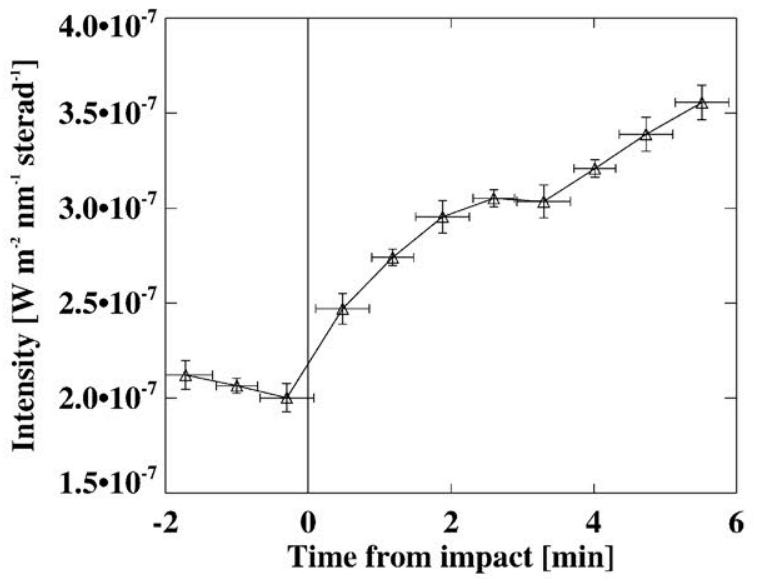

Fig. 7. Flux from Comet Tempel 1 recorded from 2 min before the impact to $4 \mathrm{~min}$ after the impact in an aperture with a radius of 2 pixels $(3000 \mathrm{~km})$. Data from both the orange filter and the clear filter are used. The clear filter images are scaled. The horizontal bar for each data point represents the exposure time.

for a particle of given radius taking into account the physics of the impact or the dust-gas interaction in the inner coma calculated with empirical relationships. Finally, we fit simultaneously the 5 images taken into account by a linear combination of all the synthetic images which fulfill the above constraint. This analysis allows us to retrieve a single size and velocity distribution which simultaneously fits all the images by comparing them with synthetic images created by the Monte Carlo program.

We considered only olivine dust particles with a density of $3780 \mathrm{~kg} \mathrm{~m}^{-3}$. Jorda et al. (this issue) describe in details the

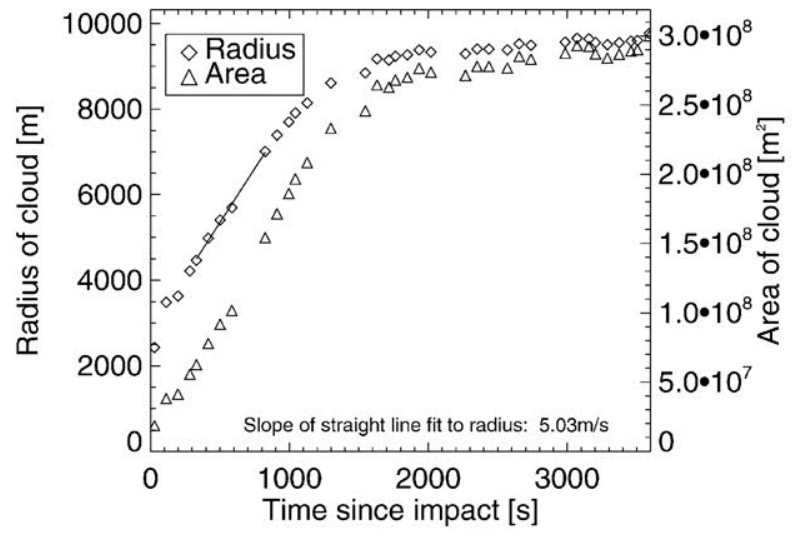

Fig. 8. Light curve in the orange filter converted to total particle cross section corresponding to measured flux assuming an albedo of 0.1 . Also given is the radius of a circle with that cross section. The slope of $5 \mathrm{~m} \mathrm{~s}^{-1}$ between 5 and 15 min corresponds to the particle velocity necessary for the brightness increase under optically thick conditions. Should the impact cloud become transparent, velocities need to be faster. For further explanation see text.

method used to retrieve the physical parameters from the images and the parameters used in the simulation.

We derived the slope of the differential dust size distributions for grains smaller than $20 \mu \mathrm{m}$ and found a power law exponent of $-3.3 \pm 0.2$. This value is consistent (within the error bars) with slopes derived recently for active comets, e.g., by Epifani et al. (2001), Fulle (2000) and Fulle et al. (1997). The velocity distribution is extremely well constrained by the images. We find a broad velocity distribution with a peak at $190 \mathrm{~m} \mathrm{~s}^{-1}$ and a FWHM of $150 \mathrm{~m} \mathrm{~s}^{-1}$. Grains with velocities of up to $600 \mathrm{~m} \mathrm{~s}^{-1}$ are clearly detected. 


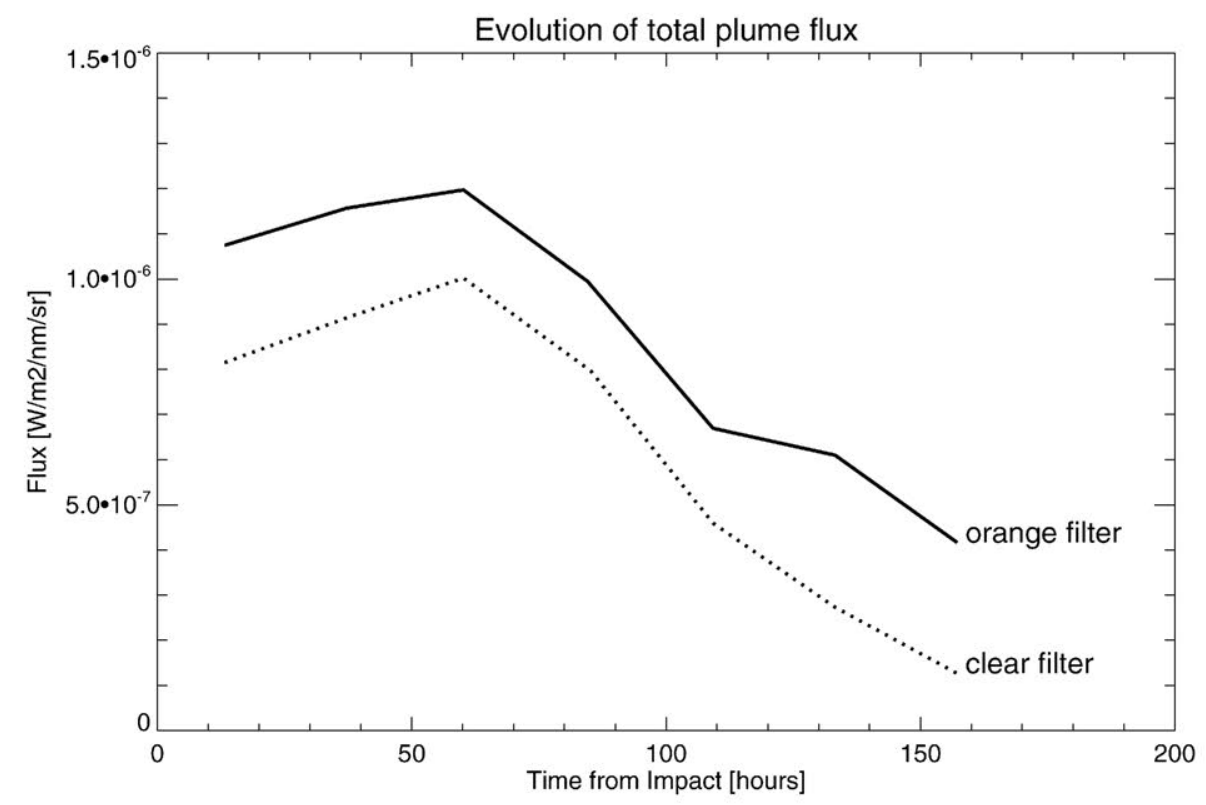

Fig. 9. Total flux by the ejecta plume through the orange and clear filter as a function of time after the impact.

The image analysis allows us to measure the mass of submicron particles. We find that particles with a radius $a<1.4 \mu \mathrm{m}$ carry a mass of only $1.6 \pm 0.2 \times 10^{5} \mathrm{~kg}$. These particles represent however more than $95 \%$ of the dust cross section. For dust particles with radii $a<100 \mu \mathrm{m}$, we find solutions with similar residuals which correspond to a mass in the range $1-14 \times 10^{6} \mathrm{~kg}$. For mass estimates smaller than $5 \times 10^{6} \mathrm{~kg}$, the kinetic energy carried by the dust is still a small fraction of the kinetic energy of the impactor.

We cannot estimate the total mass because particles larger than about $100 \mu \mathrm{m}$ are not detectable on our visible images. Therefore, the above mass estimates are likely to be only a small fraction of the total mass of particles excavated during the impact, as already pointed out by Küppers et al. (2005) from measurements of the dust cross section.

\subsubsection{Dust color}

We have estimated the reddening of the coma from the fluxes measured through the orange $(647.5 \mathrm{~nm})$ and near-IR $(882 \mathrm{~nm})$ filters. In order to avoid the influence of the contribution from gas as well as possible, we constrained our analysis to the flux measured in the innermost coma. We used circular apertures with radii between $3000 \mathrm{~km} \mathrm{(2} \mathrm{px)} \mathrm{and} 7500 \mathrm{~km}(5 \mathrm{px})$ centered on the nucleus. Constraining our analysis to this comparatively small region, we also minimized the effect of cosmic rays, numerous in our images, especially in near-IR images (due to their long exposure time).

Reddening or reflectivity gradient $\left(S^{\prime}\right.$, in percent of change per $100 \mathrm{~nm}$ ) is estimated from the reflectivity $(S)$ at the two effective wavelengths of the filters as

$$
\begin{aligned}
S^{\prime}= & 2 \times\left(S_{\mathrm{Nir}}-S_{\mathrm{Or}}\right) /\left(\left(S_{\mathrm{Or}}+S_{\mathrm{Nir}}\right) \times(882-647.5)\right) \\
& \times 10,000 .
\end{aligned}
$$

These reflectivities are calculated by dividing the extracted flux by the solar spectrum taken from Burlov-Vasiljev et al. (1995, 1998), as seen through the aforementioned filters.

The evolution of the reddening as a function of time is shown in Fig. 10. Our analysis covers from the time from -130 up to $240 \mathrm{~h}$ after the impact, when all the ejecta material has been swept out of the considered region. In this period, we have no color information from 0 up to $+3 \mathrm{~h}$, as in this period we alternatively observe with clear and orange filters in order to closely watch the effect of the impact.

From pre-impact data, we see a moderate reddening with a mean value around $7 \% / 100 \mathrm{~nm}$ in the range from 647.5 up to $882 \mathrm{~nm}$, at the phase angle of $69^{\circ}$. The actual reddening of the dust may be a few percent higher due to the stronger contribution of gas emissions to the orange filter compared to the near-IR filter. In Fig. 10, it can be seen that this slope remains constant over time with no significant change after the impact. The interpretation could be that the ejecta do not introduce significant changes on the size distribution and/or overall properties (composition, structure, etc.) of dust grains forming the 'normal' coma. Nevertheless, as our study is limited to the visible and does not cover the first $3 \mathrm{~h}$ after the impact, other scenarios are also possible. Ejecta could have an excess of large particles not contributing to the reflected light in the visible. In addition, excess of small and fast particles emitted immediately after the impact could be out of our region of study after the first $3 \mathrm{~h}$.

\subsection{Investigation of the gas content of the coma and the impact ejecta with the WAC}

The images obtained with the WAC allow deriving the production of water (from observations of $\mathrm{OH}$ and $\mathrm{OI}$ ) and $\mathrm{CN}$ in the coma before and after impact as well as the content of water and $\mathrm{CN}$ in the impact ejecta. In Section 3.2.1 an update of 


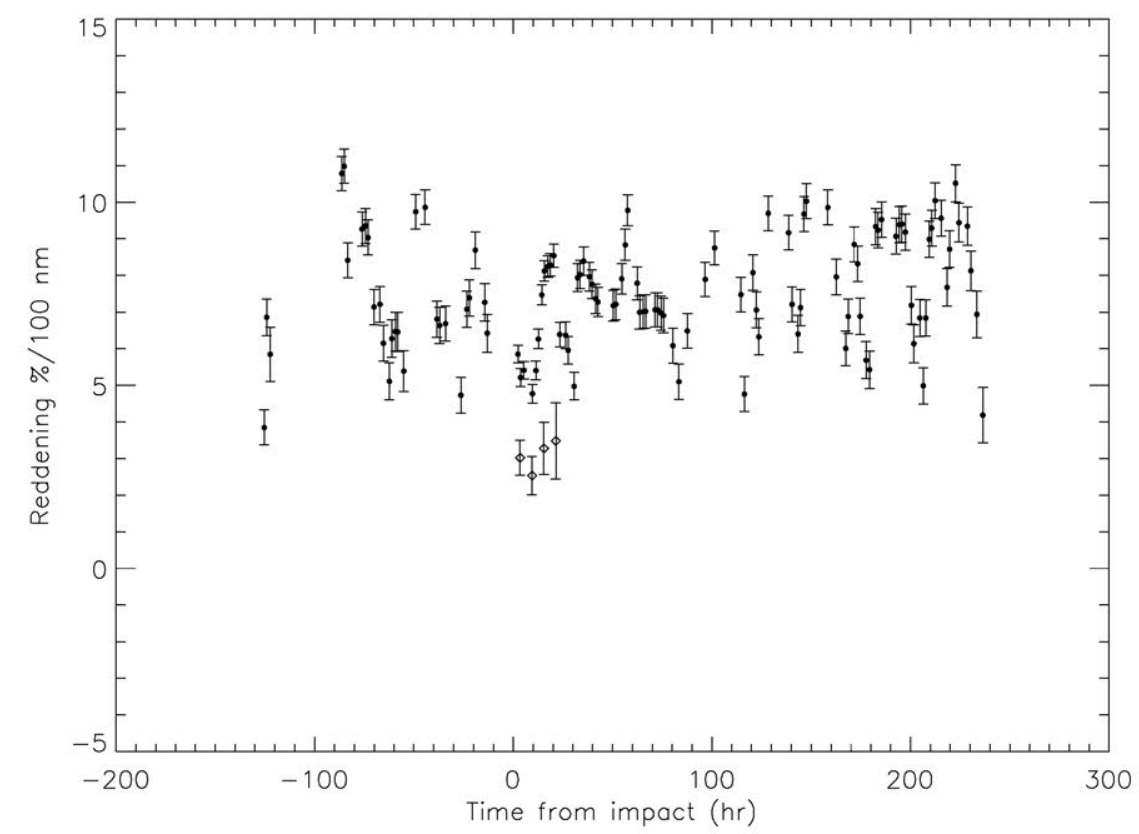

Fig. 10. Reddening of the cometary coma derived from images taken with the orange ( $648 \mathrm{~nm})$ and the near-IR $(882 \mathrm{~nm})$ filter. The open symbols show the color of the impact ejecta after subtraction of the pre-impact coma.

the first results presented by Küppers et al. (2005) and Keller et al. (2005) is given.

Sodium D-line emission could not be detected. No sodium was found in the pre- and post-impact coma. Some enhancement is seen in two images taken in the first hours after the impact, but it is currently unclear if this is due to sodium D-line emission or due to an artifact of the continuum subtraction.

\subsubsection{Water and $C N$ production}

First results about the water and $\mathrm{CN}$ production of Comet Tempel 1 before the impact and about the mass of water and the abundance of the $\mathrm{CN}$ parent in the impact cloud have been presented by Küppers et al. (2005) and Keller et al. (2005). Here we describe the data in more detail and expand and update our analysis.

The light curves of the $\mathrm{OH}$ and $\mathrm{CN}$ emission are shown in Fig. 11. The dominant time variation is caused by the impact. First a brightness increase is seen with some delay due to the photo dissociation timescale of the parent molecules of $\mathrm{OH}$ and $\mathrm{CN}$. The subsequent decrease is caused by both dissociation of $\mathrm{OH}$ and $\mathrm{CN}$ and by the molecules leaving the synthetic apertures.

Apart from the impact, very little brightness variations are seen. The standard deviation of the pre-impact data for the different apertures shown in Fig. 10 is $4-6 \%$ for $\mathrm{OH}$ and $3-4 \%$ for $\mathrm{CN}$. This is different from variations in the $\mathrm{CN}$ brightness of the order of $30 \%$ associated with the rotation of the nucleus reported by Jehin et al. (2006). We see the cause of the discrepancy in the different sizes of the fields of view: Since the gas will leave the small slit used by the Jehin et al. (2006) observations within a few hours after sublimation from the nucleus, the parent molecules of most of the $\mathrm{CN}$ they see left the nucleus relatively shortly before the observation. On the other hand, our wide angle data effectively see an average of the gas produc- tion over a time scale of tens of hours (for both the dissociation timescale of the parent molecule and the time the $\mathrm{CN}$ needs to expand outside the apertures), comparable to the $\sim 40 \mathrm{~h}$ rotation period of the comet. Much of the rotational variation of the brightness is averaged out in this way, resulting in the flat pre-impact light curves seen in Fig. 11.

We briefly summarize the method to determine the gas production, already described in Küppers et al. (2005): The fluxes from the comet are expressed in Watts and divided by the appropriate fluorescence efficiency ( $g$-factors) to convert them to numbers of molecules. Next we determine the production rate of the nominal coma (undisturbed by the impact) before and more than $150 \mathrm{~h}$ after the impact using a standard Haser model (Haser, 1957; Swamy, 2000). The Haser model assumes radial outflow with a constant velocity of both parent and daughter species. The parameters of the Haser model are the production rate of the parent species, and the lifetimes $\tau$ (or scalelength $s=\tau v$ ) of parent and daughter molecules. The expansion velocity $v$ of the daughter molecule needs to be prescribed. Because of the low signal/noise of our observations, we fit only the production rate and take published values for the lifetimes. An overview of the model parameters is given in Table 6.

For the ejecta cloud, many of the assumptions of the Haser model are not needed. In particular, the number of molecules created in the impact can be determined independent of the outflow velocity. We assume that all parent molecules are created instantaneously at the time of impact and subtract the median number of molecules measured pre-impact from the data. Then as long as the cloud of ejected material has not left a certain aperture around the nucleus, the number of molecules seen is given by

$N_{\mathrm{P}}(t)=N_{\mathrm{P}}(0) e^{-t / \tau_{\mathrm{P}}}$ 



Fig. 11. Brightness of $\mathrm{OH}$ and $\mathrm{CN}$ emission as a function of time relative to impact. The radii of the apertures with $1-5$ binned pixels corresponds to 31,200-156,000 km. About $80 \mathrm{~h}$ after the impact the noise starts to increase because the solar elongation of Tempel 1 as seen by Rosetta decreases below 90 deg. The sharp peak seen for the $\mathrm{CN}$ emission approximately $183 \mathrm{~h}$ after the impact is an artifact due to cosmic ray events.

Table 6

Parameters used in determination of gas production rates

\begin{tabular}{lllllll}
\hline Molecule & Parent & $\begin{array}{l}\text { Fluorescence efficiency } \\
{[\mathrm{W} \text { molecule }]^{\mathrm{a}}}\end{array}$ & $\begin{array}{l}\text { Parent } \\
\text { lifetime }[\mathrm{s}]^{\mathrm{a}}\end{array}$ & $\begin{array}{l}\text { Daughter } \\
\text { lifetime }[\mathrm{s}]^{\mathrm{a}}\end{array}$ & $\begin{array}{l}\text { Parent velocity } \\
v_{\mathrm{P}}\left[\mathrm{km} \mathrm{s}^{-1}\right]\end{array}$ & $\begin{array}{l}\text { Excess velocity } \\
v_{\mathrm{e}}\left[\mathrm{km} \mathrm{s}^{-1}\right]^{\mathrm{b}}\end{array}$ \\
\hline $\mathrm{OH}$ & $\mathrm{H}_{2} \mathrm{O}$ & $1.5 \times 10^{-22 \mathrm{~d}}$ & $76,750^{\mathrm{f}}$ & $131,500^{\mathrm{f}}$ & $0.7^{\mathrm{i}}$ & $f_{\mathrm{D}}^{\mathrm{c}}$ \\
$\mathrm{CN}$ & Unknown & $2.1 \times 10^{-20 \mathrm{e}}$ & $55,000^{\mathrm{g}}$ & $350,000^{\mathrm{h}}$ & $0.7^{\mathrm{i}}$ & $1^{\mathrm{i}}$ \\
\hline
\end{tabular}

a All fluorescence efficiencies and lifetimes are for $1 \mathrm{AU}$.

b Excess velocity of daughter molecule due to dissociation of parent. The expansion velocity of the daughter molecule is given by $v_{\mathrm{D}}^{2}=v_{\mathrm{P}}^{2}+v_{\mathrm{e}}^{2}$.

c Fraction of parent molecule which dissociates into daughter.

d Schleicher and A'Hearn (1988).

e Festou and Zucconi (1984).

f Budzien et al. (1994).

g The lifetime of the CN parent is uncertain (see, e.g., Rauer et al., 2003). We used a lifetime which agrees with both pre-impact data and data from the impact ejecta cloud.

h Rauer et al. (2003).

i Combi et al. (2004).

j Fray et al. (2005) for $\mathrm{HCN}$ as a parent molecule.

k Huebner et al. (1992).

and

$$
\begin{aligned}
N_{\mathrm{D}}(t) & =f_{\mathrm{D}} \times \int_{0}^{t}-\frac{\mathrm{d} N_{\mathrm{P}}}{\mathrm{d} t^{\prime}} \exp \left(-\left(t-t^{\prime}\right) / \tau_{\mathrm{D}}\right) \mathrm{d} t^{\prime} \\
& =f_{\mathrm{D}} N_{\mathrm{P}}(0) \frac{\tau_{\mathrm{D}}}{\tau_{\mathrm{D}}-\tau_{\mathrm{P}}}\left(\exp \left(-t / \tau_{\mathrm{D}}\right)-\exp \left(-t / \tau_{\mathrm{P}}\right)\right)
\end{aligned}
$$

for $\tau_{\mathrm{D}} \neq \tau_{\mathrm{P}}$.

Here $N(t)$ is the number of molecules at time $t$ and $\tau$ the lifetime. The subscripts $\mathrm{P}$ and D refer to parent and daughter molecules, respectively. $f_{\mathrm{D}}$ is the fraction of the parent molecules which decay into the daughter species. The relation does not depend on the outflow velocity of the molecules and is valid until $t=r / v$, with $r$ being the radius of the aperture and $v$ the outflow velocity.

Since publication of our first results, we have updated our analysis in several respects:
- The intensity calibration of the instrument has been revised on the basis of in flight observations of calibrated standard stars, resulting in an $\sim 30 \%$ increase in the fluxes for $\mathrm{OH}$ and an $\sim 50 \%$ increase for $\mathrm{CN}$.

- The removal of cosmic ray events and stars has been improved.

- We now include unbinned data in addition to the images with binning over $4 \times 4$ pixels in the analysis, hence doubling the size of the data.

- Before applying the Haser model, we use the model of Combi and Delsemme (1980) to convert physical scalelengths $s=\tau v$ into Haser scalelengths. This corrects for the incorrect assumption of radial outflow of the daughter molecule.

The production rates from the nominal coma of the comet and the number of molecules created from the impact ejecta are 
Table 7

Production rates of water and $\mathrm{CN}$ as determined by OSIRIS

\begin{tabular}{|c|c|c|c|c|}
\hline \multirow[t]{2}{*}{ Molecule } & \multirow[t]{2}{*}{ Binning } & \multicolumn{2}{|c|}{ Production rate $\left[\mathrm{s}^{-1}\right]$} & \multirow{2}{*}{ 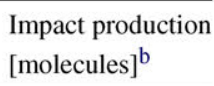 } \\
\hline & & Pre-impact & Post-impact $^{a}$ & \\
\hline$\overline{\mathrm{OH}}$ & 4 & $4.4 \times 10^{27}$ & $4.2 \times 10^{27}$ & $1.5-3 \times 10^{32}$ \\
\hline $\mathrm{OH}$ & 1 & $4.2 \times 10^{27}$ & $4.5 \times 10^{27}$ & _c \\
\hline $\mathrm{CN}$ & 4 & $1.0 \times 10^{25}$ & $1.0 \times 10^{25}$ & $6-9 \times 10^{29}$ \\
\hline $\mathrm{CN}$ & 1 & $1.1 \times 10^{25}$ & $1.2 \times 10^{25}$ & $5-8 \times 10^{29}$ \\
\hline
\end{tabular}

a Post-impact values determined at $>150 \mathrm{~h}$ when the effect of the impact is considered negligible.

b Number of molecules produced by the impact. Value does not depend on velocity.

c Signal/noise insufficient for a reliable determination.

given in Table 7. Our production rates agree well with determinations from ground-based measurements (Schleicher et al., 2006).

The number of water molecules created by the impact given in Table 7 corresponds to a mass of $(4.5-9.0) \times 10^{6} \mathrm{~kg}$, increasing the estimate from Küppers et al. (2005) by $50 \%$. If we assume that the ice in the ejecta is mostly water and that the dust/ice mass ratio is between 1 and 10 , the total mass ejected from the comet is $10^{7}-10^{8} \mathrm{~kg}$. For a density of $500 \mathrm{~kg} \mathrm{~m}^{-3}$ and a bowl-shaped crater with a diameter/depth ratio of 4 , the diameter of an impact crater excavating $10^{7}-10^{8} \mathrm{~kg}$ would be between 60 and $120 \mathrm{~m}$, within the expected range for a crater created in a regime of low or moderate (up to a few $\mathrm{kPa}$ ) strength (Schultz et al., 2005). This suggests that a substantial fraction of the impact ejecta reached a velocity above the escape velocity of the comet. On the other hand, scaling laws predict that most of the ejecta will reach less than $1 \mathrm{~m} \mathrm{~s}^{-1}$ for an impact on Tempel 1 in the gravity regime (Housen et al., 1983). The discrepancy can be resolved either if the surface material of the comet possesses some strength or if the material was quickly accelerated by collisions with cometary gas.

Our measurements of the OI red line at $630 \mathrm{~nm}$ provide an independent estimate of the water production in Comet Tempel 1 . However, the data are of lower signal/noise than the $\mathrm{OH}$ observations, and the analysis is in progress.

The ratio between the production of $\mathrm{CN}$ and water in Comet Tempel 1 before the impact was $(2.5 \pm 0.3) \times 10^{-3}$ if we take a lifetime of 55,000 s for the $\mathrm{CN}$ parent. For the impact ejecta, the $\mathrm{CN} /$ water ratio is $3.1_{-1.1}^{+2.2} \times 10^{-3}$, showing marginal evidence for a higher $\mathrm{CN} /$ water ratio than for the normal activity of the comet. As detailed in Keller et al. (2005), the emission from the pre-impact coma is more consistent with a lower lifetime of the $\mathrm{CN}$ parent $(\sim 20,000 \mathrm{~s})$, while the impact ejecta are best fit by a lifetime similar to that of $\mathrm{HCN}(\sim 70,000 \mathrm{~s})$. If the lifetimes would indeed be different, the increase in the $\mathrm{CN} / \mathrm{H}_{2} \mathrm{O}$ ratio after the impact would be larger.

A higher $\mathrm{CN} /$ water ratio in the impact ejecta compared to the background coma would be consistent with the findings from A'Hearn et al. (2005) that the ratio between the abundance of organic material and that of water was enhanced in the impact ejecta. Either the interior of the comet is compositionally different from the surface, or the more energetic impact evaporated more refractory material from the dust that is not liberated during normal activity.

\section{Discussion and conclusion}

The observations with the two OSIRIS cameras provide important clues on the events that followed the impact of the Deep Impact projectile on Comet Tempel 1. The large amount of material ejected from the comet and the velocities of the dust seen in the light curve in the first minutes after the impact suggest early acceleration of the ejected material close to the cometary surface. The most likely acceleration process is from collisions with freshly created gas molecules, either from the background coma or from the impact ejecta.

A simple model calculation can demonstrate the role of the gas liberated in an extended source for the dust motion. Fig. 12 shows the result of 1D dusty gas-dynamic calculations (Knollenberg, 1994) in spherical symmetry for the terminal velocities dust grains of different sizes would reach due to acceleration by the additional gas release of 6750 tons triggered by the impact. To model the effects of the impact generated gas cloud in a simplified way the total mass was converted to an equivalent constant production rate on a nucleus by dividing the gas mass by the release time of $40 \mathrm{~min}$ (as indicated by the lightcurve) and a surface area on a spherical nucleus corresponding to an opening angle of $120^{\circ}$ (as a reasonable assumption for the angular extent of the ejecta cloud). The time-dependent computation was stopped after the assumed release time to take the finite duration of the gas sublimation process into account. To simulate the effect that the extended source might become active with some delay after ejection (e.g., caused by the optical properties of the icy grains, fragmentation or optical thickness effects) the terminal dust velocity was computed for 4 different "virtual" radii of $R=3,6,10$, and $20 \mathrm{~km}$ which stand for different starting points of fragmentation. To be compatible with the radiation pressure calculations presented earlier the dust was again modeled as spherical grains

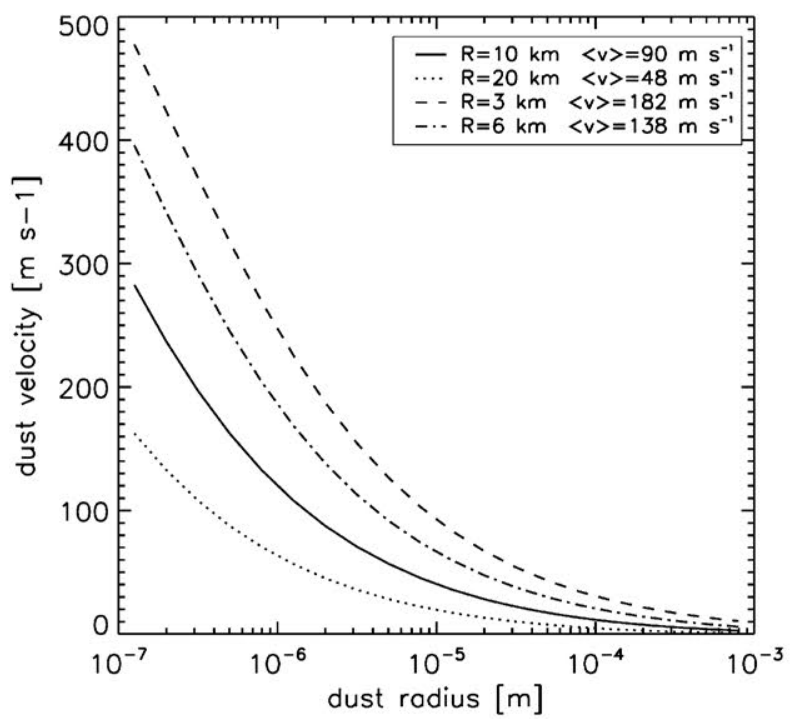

Fig. 12. Velocities of dust particles as a function of grain radius. 
with a bulk density of $3780 \mathrm{~kg} \mathrm{~m}^{-3}$. A modified exponential dust size distribution was chosen after Newburn and Spinrad (1985) with $M=2$ and an exponent of $N=3.3$. The minimum and maximum radii were $0.1 \mu \mathrm{m}$ and $1 \mathrm{~mm}$, respectively. The plot demonstrates that the acceleration of the dust grains has to take place in the vicinity of the nucleus (inside the first 2-4 nuclear radii) to be effective enough to accelerate the majority of the grains to the required outflow velocities as measured by OSIRIS. The cross section weighted velocities decrease from $\langle v\rangle=182 \mathrm{~ms}^{-1}$ for a virtual nucleus radius of $3 \mathrm{~km}$ (e.g., the acceleration from the extended source starts directly above the surface) to $\langle v\rangle=48 \mathrm{~m} \mathrm{~s}^{-1}$ for $R=20 \mathrm{~km}$. In case the effective release would start at a distance of more then 10 nuclear radii even very small grains of $0.1-\mu \mathrm{m}$ radius would not reach the observed $200 \mathrm{~m} \mathrm{~s}^{-1}$. Choosing different dust size distributions and mass loadings does not change the velocities of the dust size classes very much as long as the mass remains dominated by the large particles (e.g., $N<4$ ). It should also be noted that even the tenuous background flow of the comet, assumed to be homogeneously distributed over the dayside hemisphere, is able to accelerate particles of sizes between 1 and $100 \mu \mathrm{m}$ to velocities of some $10 \mathrm{~m} \mathrm{~s}^{-1}$ and that the critical radius for particle lift-off is about $3 \mathrm{~mm}$. Thus, particles smaller than $3 \mathrm{~mm}$ could not fall back onto the nucleus, which suggests that most of the excavated mass contributes to the ejecta cloud.

The acceleration of a significant amount of the impact ejecta to the measured high velocities at an early stage implies that most of the ejecta do not follow ballistic trajectories, instead their trajectories are strongly influenced by the motion of the surrounding gas. For this reason, care needs to be taken when drawing clues on the density and strength of the comet by interpreting the DI images in terms of classical ballistic ejection of particles (e.g., A'Hearn et al., 2005). Nucleus density and strength can be constrained by the measurements of the DI spacecraft only if there is a dust component which is largely unaffected by gas drag.

The impact liberated some non-volatile material which is not present in the normal cometary coma. This is most obvious in the spectra taken by the Deep Impact spacecraft before and after the impact (A'Hearn et al., 2005). This is confirmed in our observations of the complete ejecta cloud. The $\mathrm{CN} /$ water ratio may be higher than before the impact, possibly suggesting additional production of $\mathrm{CN}$ from the cometary dust.

The light curves seen by OSIRIS and ground-based observers (Meech et al., 2005) show that the ejecta cloud reached its final brightness after $\sim 40 \mathrm{~min}$. At that point, it was optically thin and the fragmentation of icy grains in the ejecta had mostly finished. OSIRIS monitored the activity of the comet for 10 days after the impact and an increased activity of Tempel 1 on a timescale of days (as reported by Willingale et al., 2006) can be excluded. Brightness variations unrelated to the sharp increase in the first hour after the Deep Impact event are a $~ 10 \%$ during the 2 weeks observing period and a $3 \%$ variation associated with the rotation period of Tempel 1 of $\sim 41 \mathrm{~h}$.

The final velocity of the dust ejected by the impact was around $200 \mathrm{~m} \mathrm{~s}^{-1}$. In the following days, the impact created cloud was accelerated in anti-solar direction by solar radiation pressure. The evolution of the morphology of the cloud with time allows constraining the size-velocity distribution of the dust (Jorda et al., this issue). While the velocity distribution is well constrained, the amount of large dust particles in the ejecta is uncertain, in qualitative agreement with a ground-based study by Schleicher et al. (2006).

There was no detectable long-term effect of the impact. The ejecta cloud had dispersed after about a week, no new coma structure was detected after the impact. As pointed out by Küppers et al. (2005), this suggests that impacts are not the cause of cometary outbursts or splittings. On the other hand, the surface area covered by the crater is approximately two orders of magnitudes smaller than the total active area on Tempel 1 before impact. The additional flux from a new active area would escape detection by OSIRIS

\section{Acknowledgments}

The OSIRIS imaging system on board Rosetta is managed by the Max-Planck-Institute for Solar System Research in Katlenburg-Lindau (Germany), thanks to an International collaboration between Germany, France, Italy, Spain, and Sweden. We acknowledge the work of the Rosetta Science Operations Centre at ESA/ESTEC and the Rosetta Mission Operations Centre at ESA/ESOC in coordinating the observation timelines and operating the spacecraft, in particular K. Wirth, V. Dhiri, P. Ferri, E. Montagnon, A. Hubault, J. Morales, V. Companys, and M. Lauer. We acknowledge the funding of the national space agencies ASI, CNES, DLR, the Spanish Space Program (Ministerio de Educacion y Ciencia), SNSB and ESA. IRAF is distributed by the National Optical Astronomy Observatories, which is operated by the Association of Universities for Research in Astronomy, Inc. (AURA) under cooperative agreement with the National Science Foundation. We acknowledge JPL's Horizons online ephemeris generator for providing the comet's position and rate of motion during the observations. This research has made use of NASA's Astrophysics Data System.

\section{References}

A'Hearn, M.F., Schleicher, D.G., Feldman, P.D., Millis, R.L., Thompson, D.T., 1984. Comet Bowell 1980b. Astron J. 89, 579-591.

A'Hearn, M.F., and 31 colleagues, 2005. Deep Impact: Excavating Comet Tempel 1. Science 310, 258-264.

Belton, M.J.S., and 15 colleagues, 2005. Deep Impact: Working properties for the target nucleus Comet 9P/Tempel 1. Space Sci. Rev. 117, 137-160.

Belton, M.J.S., Thomas, P.C., Carcich, B., Crockett, C.J., and Deep Impact Science Team, 2006. The spin state of 9P/Tempel 1. Lunar Planet. Sci. XXXVII. Abstract 1487.

Budzien, S.A., Festou, M.C., Feldman, P.D., 1994. Solar flux variability and the lifetimes of cometary $\mathrm{H}_{2} \mathrm{O}$ and $\mathrm{OH}$. Icarus 164, 164-188.

Burlov-Vasiljev, K.A., Gurtovenko, E.A., Matvejev, Yu.B., 1995. New absolute measurements of the solar spectrum. Solar Phys. 157, 51-73.

Burlov-Vasiljev, K.A., Matvejev, Yu.B., Vasiljeva, I.E., 1998. New measurements of the solar disk-center spectral intensity in the near IR from $645 \mathrm{~nm}$ to $1070 \mathrm{~nm}$. Solar Phys. 177, 25-40.

Combi, M.R., Delsemme, A.H., 1980. Neutral cometary atmospheres. I. An average random walk model for photodissociation in comets. Astrophys. J. 237, 633-640. 
Combi, M.R., Harris, W.M., Smyth, W.H., 2004. Gas dynamics and kinetics in the cometary coma: Theory and observations. In: Festou, M.C., Keller, H.U., Weaver, H.A. (Eds.), Comets II. Univ. of Arizona Press, Tucson, pp. 523-552.

Croft, S.K., 1980. Cratering flow fields: Implications for the excavation and transient expansion stages of crater formation. Proc. Lunar Sci. Conf. 11, $2347-2378$

Epifani, E., Colangeli, L., Fulle, M., Brucato, J.R., Bussoletti, E., de Sanctis, M.C., Mennella, V., Palomba, E., Palumbo, P., Rotundi, A., 2001. ISOCAM images of Comets 103P/Hartley 2 and 2P/Encke. Icarus 149, 339-350.

Festou, M.C., Zucconi, J.-M., 1984. CN, C2, and $\mathrm{C}_{3}$ excitation rates to be employed for interpreting photometric observations of comets. Astron. Astrophys. 134, L4-L6.

Fray, N., Bénilan, Y., Cottin, H., Gazeau, M.-C., Crovisier, J., 2005. The origin of the $\mathrm{CN}$ radical in comets: A review from observations and models. Planet. Space Sci. 53, 1243-1262.

Fulle, M., 2000. The dust environment of Comet 46/P Wirtanen at perihelion: A period of decreasing activity? Icarus 145, 239-251.

Fulle, M., Mikuz, H., Bosio, S., 1997. Dust environment of Comet Huyakutake 1996 B2. Astron. Astrophys. 324, 1197-1205.

Harker, D.E., Woodward, C.E., Wooden, D.H., 2005. The dust grains from 9P/Tempel 1 before and after the encounter with Deep Impact. Science 310 , $278-280$.

Haser, L., 1957. Distribution d'intensité dans la tête d'une comète. Bull. Acad. R. Belgique (Liège) 43, 740-750. In French.

Holsapple, K.A., Housen, K.R., 2006. Gravity or strength? An interpretation of the Deep Impact experiment. Lunar Planet Sci. XXXVII. Abstract 1068.

Housen, K.R., Schmidt, R.M., Holsapple, K.A., 1983. Crater ejecta scaling laws-Fundamental forms based on dimensional analysis. J. Geophys. Res. 88, 2485-2499.

Huebner, W.F., Keady, J.J., Lyon, S.P., 1992. Solar photo rates for planetary atmospheres and atmospheric pollutants-Photo rate coefficients and excess. Astrophys. Space Sci. 195, 1-289.

Jehin, E., Manfroid, J., Hutsemékers, D., Cochran, A.L., Arpigny, C., Jackson, W.M., Rauer, H., Schulz, R., Zucconi, J.-M., 2006. Deep Impact: Highresolution optical spectroscopy with the ESO VLT and Keck I Telescope. Astrophys J. 641, L145-L148.

Keller, H.U., 1989. Comets-Dirty snowballs or icy dirtballs? In: Hunt, J., Guyeme, T.D. (Eds.), Proc. International Workshop on Physics and Mechanics of Cometary Materials, ESA SP-302. ESA Publications Division, ESTEC, Noordwijk, pp. 39-45.

Keller, H.U., and 11 colleagues, 2005. Deep Impact observations by OSIRIS onboard the Rosetta spacecraft. Science 310, 281-283.

Keller, H.U., and 68 colleagues, 2006. OSIRIS-The scientific camera system onboard Rosetta. Space Sci. Rev. Submitted for publication.

Knollenberg, J., 1994. Modellrechnungen zur Staubverteilung in der inneren Koma von Kometen unter spezieller Berücksichtigung der HMC-Daten der GIOTTO-Mission. Ph.D. thesis, Georg-August-Universität Göttingen. In German.

Küppers, M., and 40 colleagues, 2005. A large dust/ice ratio in the nucleus of Comet 9P/Tempel 1. Nature 437, 987-990.

Licandro, J., 2005. The Deep Impact event as seen from the Roque de Los Muchachos Observatory. ING Newslett. 10, 4-6.

Maxwell, D.E., 1977. Simple Z model of cratering, ejection, and the overturned flap. In: Roddy, D.J., Pepin, R.O., Merrill, R.B. (Eds.), Impact and Explosion Cratering. Pergamon Press, New York, pp. 1003-1008.

Meech, K.J., and 208 colleagues, 2005. Deep Impact: Observations from a worldwide Earth-based campaign. Science 310, 265-269.

Melosh, H.J., 1989. Impact Cratering: A Geologic Process. Oxford Univ. Press/ Clarendon Press/Clarendon Press, Oxford, NY.

Newburn, R.L., Spinrad, H., 1985. Spectrophotometry of seventeen comets. II. The continuum. Astron. J. 90, 2591-2608.

Rauer, H., and 12 colleagues, 2003. Long-term optical spectrophotometric monitoring of Comet C/1995 OI (Hale-Bopp). Astron. Astrophys. 397 1109-1122.

Schleicher, D.G., A'Hearn, M.F., 1988. The fluorescence of cometary OH. Astrophys. J. 331, 1058-1077.
Schleicher, D.G., Barnes, K.L., Baugh, N.F., 2006. Photometry and imaging results for Comet 9P/Tempel 1 and Deep Impact: Gas production rates, postimpact light curves, and ejecta plume morphology. Astron. J. 131, $1130-1137$.

Schultz, P.R., Ernst, C.M., Anderson, J.L.B., 2005. Expectations of crater size and photometric evolution from the Deep Impact collision. Space Sci Rev. 117, 207-239.

Sugita, S., and 22 colleagues, 2005. Subaru Telescope observations of Deep Impact. Science 310, 274-278.

Sunshine, J.M., A'Hearn, M.F., Groussin, O., Feaga, L.M., Li, J.Y., Schultz, P.H., 2006. Water ice on Tempel 1: Before, during, and after the impact event. Lunar Planet. Sci. XXXVII. Abstract 1068.

Swamy, K.S., 2000. Physics of Comets, second ed. World Scientific Series an Astronomy and Astrophysics, vol. 2. World Scientific Publishing, Singapore.

Whipple, F., 1950. A comet model. I. The acceleration of Comet Encke. Astrophys. J. 111, 375-394.

Willingale, R., O'Brien, P.T., Cowley, S.W.H., Jones, G.H., McComas, D.J., 2006. X-rays from the Deep Impact collision. In: Royal Astronomical So ciety National Astronomy Meeting 2006, Leicester, UK. Abstract D.1. 